\title{
CHARACTERIZATIONS OF LINEAR GROUPS
}

\author{
BY MICHIO SUZUKI ${ }^{1}$
}

Introduction. In his address at the International Congress of Mathematicians at Amsterdam, 1954, Brauer [3] proposed a program of studying simple groups, particularly various linear groups, by giving the structure of the centralizers of elements of order 2 . He proved among other things that if a simple group $G$ contains an element $j$ of order 2 such that the centralizer $C_{G}(j)$ is isomorphic to the centralizer of an element of order 2 in a group $L$ which is either the simple group $L_{2}(q)$ or $L_{3}(q)$ (with some restriction on $q$ ), then $G$ is in fact isomorphic to $L$, except for a few isolated exceptional cases. Thus he gave a characterization of the groups $L_{2}(q)$ or $L_{3}(q)$ in terms of the structure of the centralizer of an element of order 2. The work of Brauer was followed by a large number of investigations along the same direction. This paper is a further study in this direction of characterizing linear groups by the structure of the centralizers of involutions. Its purpose is to characterize the simple groups $L_{n}(q)$ when $q$ is a power of 2 . We shall also give a survey of known results.

1. General remarks and a survey of the known results. The problems we are interested in have an intimate bearing on the fundamental problem of classifying the finite simple groups. By a theorem of Feit-Thompson [10], a nonabelian simple group is of even order. Hence the centralizers of elements of order 2 are the subgroups of a simple group, which are always present and hopefully can be exploited.

Although almost no general proposition concerning the relationship between the structure of a simple group and the structures of the centralizers of elements of order 2 is known at present, there is some indication that the situation will be improved in the near future.

We shall consider more specific situations. One formulation of the problem will be as follows: Let $G$ be a simple group such that the centralizers of elements of order 2 satisfy a group theoretical property

Expanded and improved version of an address delivered before the Chicago meeting of the Society on April 14, 1967, by invitation of the Committee to Select Hour Speakers for Western Sectional Meetings; received by the editors June 30, 1969.

The original content of the address has been published in part [32]. Theorems proved in this paper are generalizations of the results of $[32$, IV] in higher dimensional case. The papers $[32, \mathrm{~V}, \mathrm{VI}]$ are yet unpublished, but the proofs are similar to that of [36].

${ }^{1}$ Supported in part by Air Force Office of Scientific Research Grant AF-AFOSR$1468-68$ and by NSF. 
$P$. Prove that $G$ is a simple group belonging to a particular family $F$ of simple groups. There are several works concerning this problem. We refer to [35], where the problem has been solved for

$$
P=\text { nilpotency or 2-closedness. }
$$

Gorenstein [15] solved the problem when $P$ is the property of having a normal 2-complement. A particular case was given by Glauberman [14]. In each case the particular family $F$ of simple groups has been explicitly given.

There are many challenging variations of the problem. The case when $P$ is the solvability is one of the unsolved cases. According to the result of [35], 2-closedness is the property of the centralizers of elements of order 2, which almost characterizes the family of the linear groups of low rank over fields of characteristic 2 . It would be an interesting problem to investigate what property $P$ would characterize, with some exceptions, a larger family of simple groups; for example, the family of linear groups over fields of characteristic 2. If we use a weaker assumption that $G$ contains a single element of order 2 whose centralizer satisfies the property $P$, the problem becomes harder to solve. Even the case when $P$ is the nilpotency has not been solved yet. We add a remark that, over a field of characteristic 2 , the symplectic group of four variables contains a central involution whose centralizer is nilpotent.

A still more specific situation is when the structure of the centralizer is, more or less, explicitly given. Let us assume that $G$ is a simple group and that $j$ is an element of order 2 of $G$. Set $H=C_{G}(j)$. A fundamental result here is the following theorem of Brauer and Fowler [6]. There is a function $f$ such that

$$
|G| \leqq f(|H|)
$$

where $|G|$ denotes the order of $G$. Thus, if the order of $H$ is given, there can be only a finite number of isomorphism classes of simple groups $G$, which contain an element $j$ of order 2 and $H=C_{G}(j)$. General form of the function $f$ is very crude and gives an estimate of the order of $G$ too large to be of practical value. The problem here is to determine the structure of $G$ when the structure of $H$ is given. In many cases we select a known simple group $K$ and choose an element $t$ of order 2 in $K$. Assume that $H \cong C_{K}(t)$. The expected answer is that $G$ is isomorphic to $K$. In all the cases worked out so far, the elements $t$ and $j$ are assumed to be central; that is, to lie in the center of a Sylow 2-group of $K$ and $G$, respectively. Sometimes two or more nonisomorphic simple groups have isomorphic centralizers of central 
involutions. If $A_{n}$ denotes the alternating group of degree $n$, the centralizers of central involutions of $A_{4 m}$ and $A_{4 m+1}$ are isomorphic. A recent discovery of two new simple groups, the Hall-Janko group of order 604,800 and the Higman-Janko-McKay group of order $50,232,960$ provides another case. Besides, the following pair of simple groups has isomorphic centralizers: $\left(L_{2}(7), A_{6}\right)$ and $\left(L_{3}(3)\right.$, $\left.M_{11}\right)$. Just 2 triplets $\left(A_{12}, A_{13}, S_{6}(2)\right)$ and $\left(L_{5}(2), M_{24}, H_{2}\right)$ of simple groups with isomorphic centralizers are known, and no example seems to be known when four or more simple groups have isomorphic centralizers. In the above, $H_{2}$ is the simple group of order $2^{10} 3^{35} 5^{2} 7^{3} 17$, whose possibility was announced by Held [20] and whose existence was worked out by G. Higman and verified by McKay with aid of a computer.

If the structure of $H=C_{G}(j)$ is assumed to be different from the one which appears in the known simple groups, we may obtain a contradiction. But there are some fascinating exceptional cases, which have led to the discoveries of some new sporadic simple groups (Janko [22], [24]).

The list of simple groups characterized by the structure of centralizers may be found at the end of this section.

In many cases, every member of a family of simple groups is characterized. Let $L_{n}(q)$ denote the projective special linear group of dimension $n$ over a field of characteristic 2. Thus $q$ is a power of 2: $q=2^{m}$. Let $t$ be an element of order 2 of $L_{n}(q)$ which is represented by a transvection. Denote by $H(n, q)$ the centralizer of $t$ in $L_{n}(q)$. In this paper the following theorem is proved.

THEOREM 1. Let $G$ be a simple group which contains a subgroup $H$ such that

(i) $H \cong H(n, q)$ for some $n, q \geqq 2$, and

(ii) if $z$ is a nonidentity element of the center of $H$, then $C_{G}(z)=H$.

Then $G$ is isomorphic to $L_{n}(q)$ except in the following cases: $q=2$ and $n \leqq 5$.

The cases $n \leqq 4$ have been treated ([8] $n=2,[32, \mathrm{II}] n=3,[32$, IV] $n=4)$. Held [20] considered the case $n=5$ and $q=2$. So in this paper we assume either $q>2$ or $n \geqq 6$. The exceptions are as follows: $G$ may be isomorphic to the following groups; $A_{6}$ if $n=3, A_{9}$ if $n=4$, $M_{24}$ or $H_{2}$ if $n=5$.

Nonsimple groups which satisfy the conditions of Theorem 1 can be classified.

THEOREM 2. If a nonsimple group $G$ contains a subgroup $H$ which satisfies the two conditions of Theorem 1, then one of the following holds: 
(i) The center of $H$ is a normal subgroup of $G$;

(ii) $q=2$ and $H$ has a normal complement which is an abelian group of odd order;

(iii) $G$ possesses a series of normal subgroups

$$
1 \subseteq G_{1} \subseteq G_{2} \subseteq G
$$

such that $G_{1}$ is an elementary abelian subgroup of order $q^{n-1}, G_{2} / G_{1}$ is a cyclic group of order dividing $q-1$ and $G / G_{2}$ is isomorphic to the projective general linear group $P G L(n-1, q)$. Furthermore, we have

$$
\left[G, G_{2}\right] \subseteq G_{1} \text {. }
$$

This second theorem will be proved in (4.1) and (5.4) of this paper, while the proof of Theorem 1 is completed at the end of the paper.

The proof of theorems like our Theorem 1 always begins by a consideration of possible fusions of elements of order 2. The structure of a Sylow 2-group of $G$ and the assumed simplicity will restrict the possibilities. In this process "transfer theorems" of various types and the $Z$ *-theorem of Glauberman [13] are useful. We consider the structure of the centralizer of each element of order 2, if some of them are not given as a part of assumptions. If the fusion and the structure of centralizers of elements of order 2 are determined, the order of $G$ will be computed in most cases. Usually a complete discussion of possible fusion and possible structure of the centralizers is difficult, but sometimes a partial knowledge will be sufficient. In this respect, character theory may provide useful informations.

Our proof of Theorem 1 is along the line of argument discussed above, but like corresponding proof in [32, IV] we tried to avoid the discussion of fusion and structure of centralizers, other than given, as much as possible. At the end the identification of $G$ with $L_{n}(q)$ is done by constructing a $(B, N)$-pair in $G$ and using a characterization of $L_{n}(q)$ as a group with a particular $(B, N)$-pair. For the importance and required results concerning $(B, N)$-pairs, we refer to the works of Tits [40], [41].

The structure theorems of (TI)-groups play important roles in several places of the proof. A group is called a (TI)-group if two distinct Sylow 2-groups have only the identity element in common (see [34]). Our proof is group theoretical, and the character theory is never used explicitly.

The following list is almost self-explanatory. For example, the second line means that the family of simple groups $L_{3}(q)$ for odd $q$ has been characterized by Brauer [3], [4], and when $q=3$, the Mathieu 
group $M_{11}$ has the same centralizer as $L_{3}(3)$, and it is the unique exception. We use Artin's notation [1] to denote the classical simple groups.

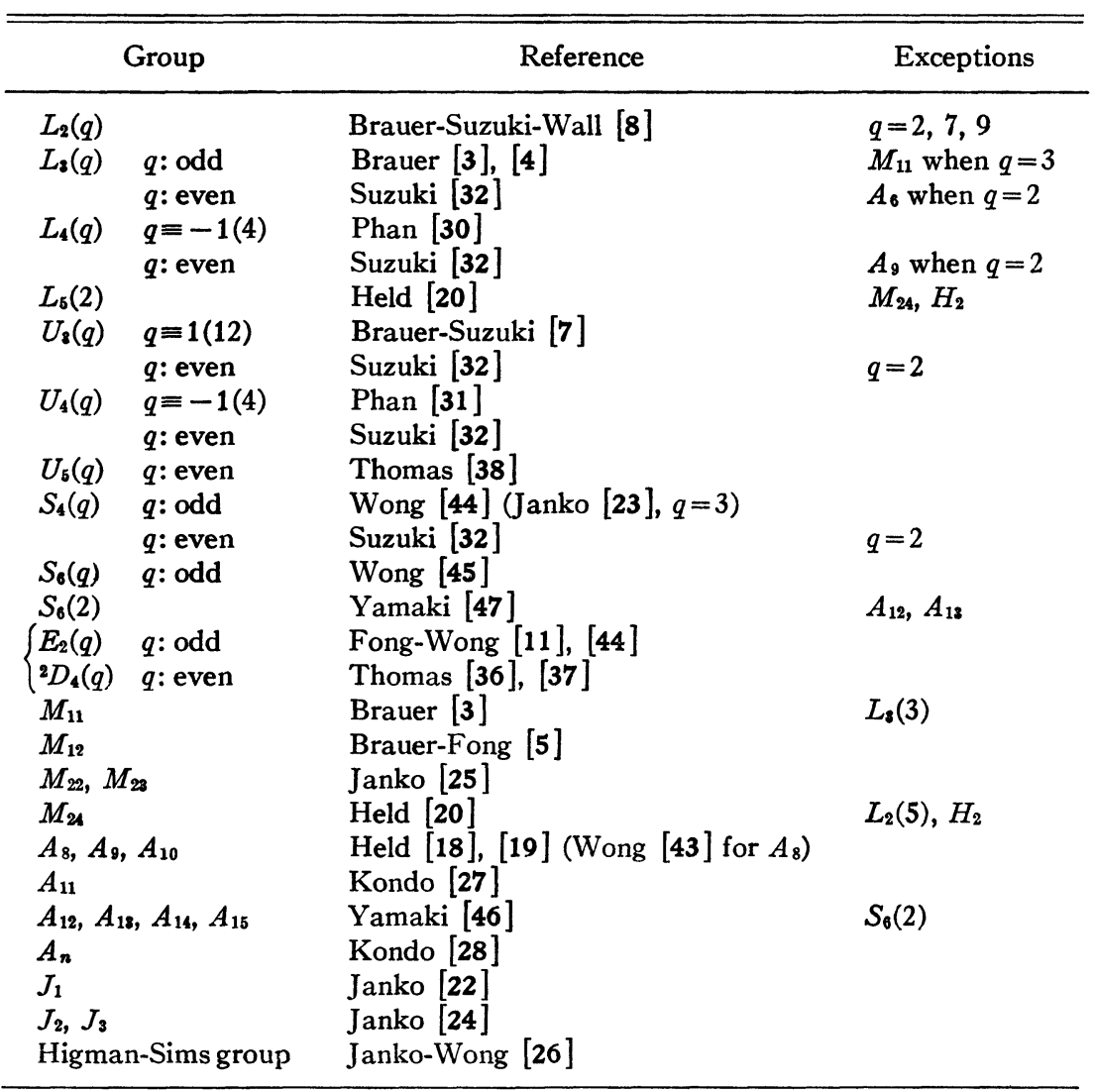

2. Notation and necessary lemmas. In the rest of this paper we shall consider only the linear group defined over a finite field of characteristic 2 . Let $F$ be the field of $q$ elements where $q=2^{m}$ is a power of 2 . We identify the elements of the general linear group $\mathrm{GL}(k, q)$ with the $k \times k$ nonsingular matrices whose entries are taken from $F$. Thus an element $x$ of $\mathrm{GL}(k, q)$ is written as

$$
x=\left(x_{i j}\right) \quad x_{i j} \in F \quad i, j=1, \cdots, k .
$$

If this element is written, for example, as 


$$
x=\left(\begin{array}{c:c}
x & \\
\hdashline y & z
\end{array}\right),
$$

it is tacitly assumed that this is a block decomposition of the matrix $x$, the letters $X, Y, Z$ are submatrices of appropriate size and the blank space represents the zero matrix. If the letter $I$ with or without suffix is used in place of $X$ ( $Y$ or $Z$ ), this explicitly means that $X$ is the identity matrix and the suffix indicates the size of $X$. The size of submatrices is allowed to be zero. If this happens, for example if the size of $Z$ is zero, the corresponding row and column, $(Y, Z)$ in the example, are to be deleted. A submatrix of size $1 \times 1$ may be identified with an element of $F$. So if, for example, the block $Z$ of the above matrix is of size $1 \times 1$, we may use the corresponding field element $z$ to denote $Z$. Conversely, any field element used in the expression of $x$ represents a block of size $(1,1)$.

(2.1) An element of order 2 of $\mathrm{GL}(k, q)$ is conjugate to an element

$$
j_{l}=\left(\begin{array}{lll}
I & & \\
& I & \\
I_{l} & & I
\end{array}\right) \quad(1 \leqq l \leqq k / 2) .
$$

Remark. A caution about our notation. The lower left corner is the $l \times l$ identity matrix. By our convention the top and bottom $I$ on the main diagonal are $I_{l}$. But the middle $I$ may be of different size. It is in fact of size $k-2 l$. If $k=2 l$, the middle row and column are to be deleted; so it is

$$
\left(\begin{array}{ll}
I & \\
I & I
\end{array}\right) .
$$

The letter $j_{l}$ denotes always the matrix defined by (2.2) in some $\mathrm{GL}(k, q)$. Since finite fields are perfect, an element of order 2 is conjugate to its canonical form $j_{l}$ in the special linear group $\operatorname{SL}(k, q)$.

(2.3) The centralizer of $j_{l}$ in $\operatorname{SL}(k, q)$ is the totality of matrices of the form

$$
\left(\begin{array}{lll}
X & & \\
P & Y & \\
Q & R & X
\end{array}\right) \quad(\operatorname{det} X)^{2} \operatorname{det} Y=1
$$


where $X$ is an $l \times l$ nonsingular matrix and $\operatorname{det} X$ is the determinant of $X$. The mapping which sends the matrix (2.4) to the pair $(X, Y)$ of the direct product of $\mathrm{GL}(l, q) \times \mathrm{GL}(k-2 l, q)$ is a homomorphism. The image of this homomorphism contains $\mathrm{SL}(l, q) \times \mathrm{SL}(k-2 l, q)$ and covers both factors $\mathrm{GL}(l, q)$ and $\mathrm{GL}(k-2 l, q)$ if $k>2 l$. The kernel is the maximal normal 2-subgroup of the centralizer.

In the following we always assume that $n \geqq 5$. By definition $L_{n}(q)$ is the factor group of $\operatorname{SL}(n, q)$ by its center. The center of $\operatorname{SL}(n, q)$ is a cyclic group of order $d=(n, q-1)$ consisting of scalar matrices, where $(n, q-1)$ is the greatest common divisor of $n$ and $q-1$. It is in particular a group of odd order.

Let $z$ be the element of order 2 of $L_{n}(q)$ which is represented by $j_{1}$ (cf. (2.2)).

(2.5) Let $H$ denote the centralizer of $z$ in $L_{n}(q)$. Then $H$ is isomorphic to the factor group of the group of the matrices (2.4) by the center of $\operatorname{SL}(n, q)$. If $O$ denotes the maximal normal 2-group of $H$, the factor group $H / O$ is isomorphic to $\mathrm{GL}(n-2, q) / C$ where $C$ is the set of scalar matrices $\lambda I_{n-2}$ with $\lambda^{n}=1$.

By (2.5), a Sylow 2-group of $H$ is isomorphic to the group of lower triangular matrices with 1 on the main diagonal. Let $S$ denote the totality of such matrices

$$
\left(a_{i j}\right) \quad \begin{cases}a_{i i}=1 & i=1, \cdots, n, \\ a_{i j}=0 & i<j .\end{cases}
$$

We identify $S$ as a Sylow 2-group of $H$.

(2.7) $N_{H}(S)$ is a semidirect product of $S$ and $D_{n-2} / C$ where $D_{n-2}$ is the totality of nonsingular diagonal matrices of degree $n-2$ and $C$ is the subgroup defined in (2.5). If $d \in D_{n-2}$ is the element with $\mu_{1}, \cdots, \mu_{n-2}$ on the main diagonal in that order, the conjugation by $d$ induces an automorphism of $S$ which sends

$$
\left(a_{i j}\right) \text { into }\left(\mu_{i-1}^{-1} \mu_{j-1} a_{i j}\right)
$$

where $\mu_{0}=\mu_{n-1}$ and $\mu_{0} \mu_{1} \cdots \mu_{n-1}=1$.

We remark that if the pair $(i, j)$ is fixed, then $\mu_{i-1}^{-1} \mu_{j-1}$ will be an arbitrary nonzero element of $F$ for a suitable choice of $\mu_{1}, \cdots, \mu_{n-2}$, unless the pair $(i, j)$ is $(n, 1)$ in which case $\mu_{i-1}^{-1} \mu_{j-1}=1$ for any choice.

Some subgroups of $S$ are important in the following discussion. We denote by (2.6) a generic element of $S$. We define the following subgroups of $S$ : 
$O$ : the maximal normal 2-group of $H$; the totality of elements of $S$ with relations $a_{i j}=0$ for all $i, j$ such that $2 \leqq j<i \leqq n-1$;

(2.8) $P$ : the subgroup of $O$ with further restrictions $a_{n j}=0$ for all $2 \leqq j<n$

$R$ : the subgroup of $O$ with $a_{i 1}=0$ for all $i$ such that $1<i<n$;

$T$ : the subgroup of $S$ consisting of all the matrices (2.6) with $a_{21}=a_{n n-1}=0$.

Furthermore set

$$
Z=P \cap R, \quad U=T P \text { and } V=T R .
$$

The structure of the group $H$ can be studied by matrix computation. By (2.5), an element $h$ of $H$ is a coset of the center of $\operatorname{SL}(n, q)$. Suppose that $h$ is represented by the matrix (2.4). The automorphism of $O$ induced by the conjugation by $h$ sends an element

$$
\left(\begin{array}{lll}
1 & & \\
A & I & \\
B & C & 1
\end{array}\right) \text { into }\left(\begin{array}{lll}
1 & & \\
A^{\prime} & I & \\
B^{\prime} & C^{\prime} & 1
\end{array}\right)
$$

where

$$
A^{\prime}=Y^{-1} A X, B^{\prime}=B+C P X^{-1}+R Y^{-1} A \text { and } C^{\prime}=X^{-1} C Y .
$$

(2.11) (i) The group $Z$ is the center of $H$.

(ii) Both $P$ and $R$ are self-centralizing abelian normal subgroup of $H$.

(iii) The center $Z(T)$ of $T$ is a subgroup of order $q^{4}$ consisting of matrices

$$
\left(\begin{array}{lll}
I & & \\
& I & \\
Q & & I
\end{array}\right)
$$

where $Q$ is an arbitrary $2 \times 2$ matrix, and

$$
Z(T)=C_{H}(T) .
$$

(iv) Let $u$ be an involution of $Z(T)$. If $u \notin O$, then $C_{S}(u)=T$. If $u \in O-Z$, then $C_{S}(u)$ is a subgroup of index $q$ of $S$. For all $u \in Z(T)$, $C_{S}(u)$ is a Sylow 2-group of $C_{H}(u)$.

(v) There are precisely 3 conjugate classes of involutions of $H$, which contain elements of $O-Z$. Each class contains an element of $Z(T)$.

(vi) $S$ is the unique Sylow 2-group of $H$ which contains $T$.

Proof. The assertions (i), (ii) and (v) follow easily from (2.10), and (iii) is proved by simple computation. Any Sylow 2-group of $H$ con- 
tains $O$ by definition of $O$. Hence a Sylow 2-group which contains $T$ must contain $T$ and $O$. Since $S=T O$, (vi) is proved. The assertion (iv) follows by computation and (vi).

(2.12) (i) Let $A$ be an elementary abelian subgroup of $S$ which is normalized by $T$. Suppose that $A$ contains a matrix $\left(a_{i j}\right)$ with $a_{21} \neq 0$. Then for any element $\left(x_{i j}\right)$ of $A, x_{i j}=0$ for $j \geqq 2$ and $j<i$ except the $2 \times 2$ submatrix in the lower left corner, where

$$
\left(\begin{array}{ll}
x_{n-12} & x_{n-1} \\
x_{n 2} & x_{n 3}
\end{array}\right)=\left(\begin{array}{ll}
\lambda x_{31} & \lambda x_{21} \\
\mu x_{31} & \mu x_{21}
\end{array}\right)
$$

for some $\lambda$ and $\mu$.

(ii) Let $A$ be an abelian subgroup of $S$ which is normalized by $T$. If $A$ is not contained in $T,|A| \leqq q^{n-1}$.

(iii) $T$ is generated by all the abelian normal sugbroups of $S$ of order at least $q^{2(n-2)}$.

Proof. First we prove that an element of $A$ is uniquely determined by the first column. Suppose by way of contradiction that there are two distinct elements $x$ and $y$ with the same first column. Let $i$ be the smallest integer such that $x$ and $y$ have different $i$ th row. We write

$$
x=\left(\begin{array}{ccc}
1 & & \\
A & B \\
C & X & 1 \\
. & . & .
\end{array}\right) \text { and } y=\left(\begin{array}{ccc}
1 & & \\
A & B & \\
C & Y & 1 \\
. & . & .
\end{array}\right)
$$

where $(C, X, 1,0)$ is the $i$ th row. We have

$$
y^{-1} x=\left(\begin{array}{ccc}
1 & & \\
& I & \\
X-Y & 1 \\
. & . & .
\end{array}\right) \in A .
$$

Since $X-Y \neq 0$ and $T$ normalizes $A$, there is an element $u$ of $A$ such that

$$
u=\left(\begin{array}{ccc}
1 & & \\
& I & \\
& U & 1 \\
. & . & .
\end{array}\right) \quad \text { with } U=(1,0, \cdots, 0)
$$


By assumption $A$ contains an element $a=\left(a_{i j}\right)$ with $a_{21} \neq 0$. The $(i, 1)$ place of the commutator $[a, u]$ has an entry $a_{21} \neq 0$. This contradicts the commutativity of $A$.

The elements of $A$ are determined by its first column. So an element $x$ of $A$ is written as

$$
x=\left(\begin{array}{ll}
1 & \\
X & U(X)
\end{array}\right)
$$

where $U(X)$ is a lower triangular matrix determined uniquely by the column vector $X$. Commutativity of $A$ yields

$$
X+U(X) Y=Y+U(Y) X
$$

for two column vectors $X$ and $Y$. If

$$
v=\left(\begin{array}{ll}
1 & \\
& V
\end{array}\right)
$$

is an element of $T$, we have

$$
v x v^{-1}=\left(\begin{array}{cc}
1 & \\
V X & V U(X) V^{-1}
\end{array}\right) .
$$

Since $T$ normalizes $A$, this yields

$$
U(V X)=V U(X) V^{-1}
$$

for any admissible $V$ for which the element $v$ belongs to $T$. In particular $U(X)$ commutes with any admissible $V$ such that $V X=X$. Let $X_{\alpha}$ be the column vector such that the top entry of $X_{\alpha}$ is $\alpha$ and the rest are zero. Then there is an element $x_{\alpha}$ of $A$ with $X=X_{\alpha}$ and (2.14) yields that entries of $U\left(X_{\alpha}\right)$ below the main diagonal are zero except the $2 \times 2$ submatrix of the lower left corner. Since the group $A$ is elementary, the first column of $U\left(X_{\alpha}\right)$ consists of zeros except the top. Thus all columns of $U\left(X_{\alpha}\right)-I$ are zero except the second, where the bottom two entries may be nonzero. Let $V_{\alpha}$ denote the last two entries of the second column of $U\left(X_{\alpha}\right)$ (considered as a two-dimensional column vector). If $Y$ is any column vector with the top entry zero, (2.14) yields that all columns of $U(Y)-I$ are zero except the first, which we denote $Y_{1}$. The equation (2.13) yields that

$$
\alpha Y_{1}=\left(\begin{array}{c}
0 \\
\beta V
\end{array}\right)
$$


where $\beta$ is the second entry from the top of $Y$. This shows that $Y_{1}$ is a function of $\beta$ only. Furthermore for some constant vector $W$, $V_{\alpha}=\alpha W$ and the nonzero entries of $Y_{1}$ are at bottom where we have $\beta W$. Since an arbitrary element of $A$ may be written as a product of the form $x_{\alpha} y$ where the column vector $Y$ corresponding to $y$ has zero at the top, the assertion (i) is proved.

The proposition (ii) follows from the first half of the above argument.

The totality of matrices of the form

$$
\left(\begin{array}{ll}
I & \\
X & I
\end{array}\right)
$$

forms an abelian normal subgroup of $S$. If $X$ is of size $(k, l)$, this abelian group is of order $q^{k l}$. Since $k+l=n, k l \geqq 2(n-2)$ unless either $k=1$ or $l=1$. Thus $T$ is generated by abelian normal subgroups of order at least $q^{2(n-2)}$. Since $2(n-2)>n-1$ for $n \geqq 4$, any abelian normal subgroup of $S$ of order at least equal to $q^{2(n-2)}$ is contained in $T$ by (ii). This proves (iii).

(2.15) A conjugate class of involutions of $\mathrm{H}-\mathrm{O}$ is represented by one of the following elements:

$$
\begin{aligned}
& \left(\begin{array}{ccccc}
1 & & & & \\
& I & & & \\
& & I & & \\
& I_{k} & & I & \\
z & & & & 1
\end{array}\right),\left(\begin{array}{llll}
1 & & & \\
& I & & \\
& & I & \\
& I_{k} & & I
\end{array}\right),\left(\begin{array}{cccc}
I & & & \\
& I & & \\
I_{k} & & I & \\
& & & 1
\end{array}\right), \\
& \left(\begin{array}{lll}
I & & \\
& I & \\
I_{k} & & I
\end{array}\right) .
\end{aligned}
$$

Proof. An element of $H$ is represented by a matrix of the form (2.4) where $Y \in \mathrm{GL}(n-2, q)$. Let $J$ be a conjugate class of involutions of $H-O$. Then $J$ contains an element $j$ which is represented by (2.4) with $Y=j_{l}$ of (2.2). By simple computation we see that $j$ is conjugate to one of the elements in (2.16). The index $k$ is equal to $l$ in the first case, $l+1$ for the next two cases and $l+2$ for the last.

(2.17) Let $j$ be one of the canonical forms in (2.16). The following hold:

(i) $C_{H}(j) \cap S$ is a Sylow 2-group of $C_{H}(j)$; 
(ii) If $j$ is the first element of (2.16), then the center of $C_{S}(j)$ contains $Z(T)$. We have

$$
\begin{aligned}
\left|Z\left(C_{S}(j)\right)\right| & =q^{5} \quad \text { if } k \geqq 2, \\
& =q^{4} \quad \text { if } k=1 ;
\end{aligned}
$$

(iii) If $j$ is the second or third element of (2.16), the order of $Z\left(C_{S}(j)\right)$ is $q^{3}$. If $j$ is the last element, $\left|Z\left(C_{S}(j)\right)\right|=q^{2}$.

This is proved by easy computation.

We need lemmas of general nature.

(2.18) Let $u$ and $v$ be two involutions in a torsion group $G$. If $u$ is not conjugate to $v$ in $G$, there exists an involution $w$ of $G$ which commutes with both $u$ and $v$. Furthermore either uw is conjugate to $u$ and vw is conjugate to $v$, or $u w$ is conjugate to $v$ and vw to $u$.

This is known. By assumption the element $u v$ has even order $2 n$. Set $w=(u v)^{n}$. If $n$ is even, $u w$ is conjugate to $u$ because

$$
u w=(v u)^{m} v u v(u v)^{m} \text { for } n=2 m+2 .
$$

Similarly $v w$ is conjugate to $v$. If $n$ is odd, $u w$ is conjugate to $v$ and $v w$ to $u$.

(2.19) Let $G$ be a finite group of even order and $S$ be a Sylow 2-group of G. Set

$$
D=\cap\left(S \cap S^{x}\right)
$$

where the intersection is taken over all those conjugate subgroups $S^{x}$ of $S$ which satisfy $S \cap S^{x} \neq 1$. Suppose that there is a Sylow 2-group $S_{0}$ of $G$ such that $S \cap S_{0}=1$. If $S$ contains more than one involution, then either $D=S$ or $D=1$.

The following proposition is an immediate consequence of (2.19).

(2.20) Let $G$ be a finite group of even order and $S$ a Sylow 2-group. Suppose that $S$ contains more than one involution and that there is a Sylow 2-group $S_{0}$ of $G$ such that $S \cap S_{0}=1$. Suppose furthermore that there is a subgroup $T$ of $S$ which satisfies the following two properties:

(1) $T \neq 1$ and

(2) for any conjugate subgroup $S^{x}$ of $S, S \cap S^{x} \neq 1$ implies that $S^{x} \supseteq T$. Then $G$ is a (TI)-group: that is, if $S^{x} \neq S$, then $S^{x} \cap S=1$.

Assume the validity of (2.19). Let $D$ be the subgroup defined in (2.19). The assumption of (2.20) implies that $D \supseteq T \neq 1$. Hence by 
(2.19), $D=S$. This means that $S \cap S^{x} \neq 1$ implies $S=S^{x}$. So $G$ is a (TI)-group.

For the properties of (TI)-groups, see [34]. We remark here that if a Sylow 2-group of a (TI)-group $G$ is not normal and contains more than one involution then $G$ contains a normal subgroup of odd index, which is isomorphic to one of the simple groups $L_{2}(q), S_{z}(q)$ or $U_{3}(q)$ for some $q$, a power of 2 .

Proof of (2.19). The subgroup $D$ defined in (2.19) is uniquely determined by $S$. So we denote it as $D=D(S)$. If $x \in G$, then $D\left(S^{x}\right)$ $=D(S)^{x}$. If $D=1$, there is nothing to prove. Thus we assume that $D \neq 1$. We prove a series of lemmas.

(a) Let $S_{1}$ be a Sylow 2-group of $G$. If $S \cap S_{1} \neq 1$, then $D\left(S_{1}\right)=D(S)$.

Proof. Since $S_{1} \cap S \neq 1, S_{1} \cap S \supseteq D=D(S)$; in particular $S_{1} \supseteq D$. Let $S^{x}$ be a Sylow 2-group such that $S \cap S^{x} \neq 1$. By defintion $S^{x} \cap S \supseteq D$. Hence $S_{1} \cap S^{x} \supseteq D$ which is not 1 by assumption. By definition of $D\left(S_{1}\right)$ we conclude that $S^{x} \supseteq D\left(S_{1}\right)$. Since $D(S)$ is the intersection of all those Sylow 2-groups $S^{x}$ which satisfy $S \cap S^{x} \neq 1, D(S) \supseteq D\left(S_{1}\right)$. So $D(S)=D\left(S_{1}\right)$ because they have the same order.

(b) Involutions of $G$ are conjugate to each other.

Proof. By assumption $G$ contains a Sylow subgroup $S_{0}$ such that $S \cap S_{0}=1$. By way of contradiction suppose that there is a pair of involutions of $G$ which are not conjugate. Then there is a pair of involutions $u$ and $v$ such that $u \in S, v \in S_{0}$ and $v$ is not conjugate to $u$. By (2.18) there is an involution $w$ which commutes with both $u$ and $v$. The subgroup $\langle w, u\rangle$ is of order 4 . There is a Sylow 2-group $S_{1}$ of $G$ which contains $\langle w, u\rangle$. Similarly there is a Sylow 2-group $S_{2}$ of $G$ which contains $\langle w, v\rangle$. We have $S \cap S_{1} \neq 1$ because $S \cap S_{1} \ni u \neq 1$. Similarly $S_{1} \cap S_{2} \neq 1$ and $S_{2} \cap S_{0} \neq 1$. By (a) we have $D(S)=D\left(S_{1}\right)$ $=D\left(S_{2}\right)=D\left(S_{0}\right)$. This contradicts the assumption $D(S) \neq 1$ because $D\left(S_{0}\right)=D(S)$ is contained in $S \cap S_{0}=1$.

(c) $D=D(S)$ contains all the involutions of $S$.

Proof. There is an involution $j$ of $D$ since $D \neq 1$. Let $u$ be an involution of $S$. By (b), there is an element $x$ of $G$ such that $u=j^{x}$. Thus $S \cap S^{x}$ contains $u$ in common. By (a) we have $D=D(S)=D\left(S^{x}\right)$ $=D(S)^{x}$. Hence $x$ normalizes $D$ and $u=j^{x} \in D$.

(d) $N_{G}(D)$ is strongly embedded (see [16], [33]).

Proof. The group $S$ is clearly contained in $N_{G}(D)$. Hence any involution of $N_{G}(D)$ is conjugate in $N_{G}(D)$ to an involution of $S$. By 
(c), $D$ contains all the involutions of $S$. Hence $D$ contains all the involutions of $N_{G}(D)$. Let $j$ be an involution of $N_{G}(D)$. If $x \in C_{G}(j)$, $S \cap S^{x}$ contains $j=j^{x}$. Hence

$$
D=D(S)=D\left(S^{x}\right)=D(S)^{x}=D^{x} .
$$

Thus $N_{G}(D) \supseteq C_{G}(j)$ for any involution $j$ of $N_{G}(D) . N_{G}(D)$ contains $N_{G}(S)$, but $N_{G}(D)$ does not contain involutions of $D\left(S_{0}\right)$. Thus $N_{G}(D)$ satisfies all the conditions of a strongly embedded subgroup.

We apply a theorem of Bender [2]. Since $G$ contains a strongly embedded subgroup and $S$ contains more than one involution, $G$ is a (TI)-group by Bender's theorem. Hence $D=S$ and the proof is finished.

3. Structure of $N_{G}(T)$ and fusion of involutions. Let $H$ be the centralizer of an involution in the center of a Sylow 2-group of $L_{n}(q)$. The structure of $H$ was discussed in (2.5). In the rest of this paper we consider a finite group $G$ which contains a subgroup isomorphic to $H$. We will identify this subgroup with $H$, so $H \subseteq G$. The basic assumptions are

(1) $G$ contains $H$ with $n \geqq 5$,

(2) if $z$ is an involution of $Z(H)$, then $C_{G}(z)=H$.

We use the same notation as in the preceeding section. So $S$ is a Sylow 2-group of $H$ and the notations (2.8) and (2.9) are used freely. In particular $Z=Z(H)$.

(3.1) (i) If $Z \cap Z^{x} \neq 1$, then $Z=Z^{x}$.

(ii) Let $X$ be a subgroup of $G$ such that $C_{G}(X) \subseteq X$. If $Z(X) \cap Z^{x} \neq 1$, then $Z^{x} \subseteq Z(X)$.

Proof. The first proposition is a particular case of (ii) where $X=H$. In order to prove (ii), choose a nonidentity element $z$ of $Z(X) \cap Z^{x}$. Then $C_{G}(z)$ contains $X$. By the basic assumption (2), $C_{G}(z)=C_{G}\left(Z^{x}\right)$. This implies that $\left[X, Z^{x}\right]=1$. Hence $Z^{x} \subseteq C_{G}(X)$. By assumption $C_{G}(X) \subseteq X$, so

$$
Z^{x} \subseteq X \cap C_{G}(X)=Z(X) .
$$

(3.2) (i) $C_{G}(T)=Z(T)$.

(ii) Let $W$ be any subgroup such that $T \subseteq W \subseteq S$. Then

$$
N_{G}(W) \subseteq N_{G}(T) .
$$

(iii) If $S_{1}$ is any Sylow 2-group of $G$ which contains $T, S_{1}$ normalizes $T: S_{1} \subseteq N_{G}(T)$. 
(iv) If $S_{1}$ is a Sylow 2-group of $N_{G}(T)$, the index $\left|S: S \cap S_{1}\right|$ is 1 , $q$ or $q^{2}$.

Proof. (i) Since $Z \subseteq T, C_{G}(T)$ is contained in $C_{G}(Z)=H$. Thus $C_{G}(T)=C_{X}(T)=Z(T)$ by (2.11) (iii).

(ii) Let $x$ be an element of $N_{G}(W)$. By (2.12) (iii) $T$ is generated by the abelian normal subgroups of $S$ of order at least $q^{2(n-2)}$. Let $A$ be an abelian normal subgroup of $S$ of order at least $q^{2(n-2)}$. Then $A \subseteq T \subseteq W$. Hence $A^{x}$ is an abelian group of order larger than $q^{n-1}$, which is normalized by $T$. By (2.12) (ii), $A^{x} \subseteq T$. This implies that $x$ normalizes $T$. Thus $N_{G}(W) \subseteq N_{G}(T)$.

(iii) Set $D=S_{1} \cap N_{G}(T)$ and assume, by way of contradiction, that $D \neq S_{1}$. By Sylow's theorem, $D$ is contained in a Sylow 2-group $S_{2}$ of $N_{G}(T)$ and $S_{2}^{x}=S$ for some element $x$ of $N_{G}(T)$. Then

$$
T \subseteq D^{x}=N_{G}(T) \cap S_{1}^{x} \subseteq S_{2}^{x}=S .
$$

By (ii), $N_{G}\left(D^{x}\right) \subseteq N_{G}(T)$. Hence

$$
D^{x} \subseteq N_{G}\left(D^{x}\right) \cap S_{1}^{x} \subseteq N_{G}(T) \cap S_{1}^{x}=D^{x} .
$$

This implies that $D^{x}$ coincides with its normalizer in $S_{1}^{x}$. This contradicts a fundamental property of a $p$-group.

(iv) Since $T \subseteq S_{1}, Z\left(S_{1}\right)=Z_{1}$ centralizes $T$. By (i), $Z_{1} \subseteq Z(T)$. Let $S_{2}$ be a Sylow 2-group of $C_{G}\left(Z_{1}\right)$ which contains $C_{S}\left(Z_{1}\right)$. Then both $S_{1}$ and $S_{2}$ are Sylow 2-groups of $C_{G}\left(Z_{1}\right)$ which contain $T$. By (2.11) (vi), $S_{2}=S_{1}$. This implies that $S \cap S_{1}=C_{S}\left(Z_{1}\right)$. If $Z_{1}$ contains an element of $Z(T)-O, C_{S}\left(Z_{1}\right)=T$ (2.11) (iv). If $Z_{1} \subseteq 0$ and if $Z_{1}$ contains an element $u$ of $O-Z$, then $C_{S}(u)$ is of index $q$ in $S$. But the assumption (2) yields that $C_{S}(u)=C_{S}\left(Z_{1}\right)$. This proves (iv).

(3.3) Both $U$ and $V$ are normal subgroups of $N_{G}(S)$.

Proof. Let $x$ be any element of $N_{G}(S)$. Then $x$ normalizes $Z=Z(S)$, $H=C_{G}(Z)$ and finally $O$, because $O$ is a characteristic subgroup of $H$. There are precisely 3 conjugate classes of involutions in $O-Z$ by (2.11) (v). The sets $P-Z$ and $R-Z$ are two of them and each contains $q^{n-1}-q$ elements. The third class contains $\left(q^{n-1}-q\right)\left(q^{n-2}-q\right)$ elements. The conjugation by $x$ induces a permutation of the conjugate classes of $H$ containing involutions of $O-Z$. If we denote this permutation as $f(x), f$ is a representation of $N_{G}(S)$ and the kernel of $f$ contains $S$. Thus the image of $f$ is a group of odd order. Since the third class contains more elements than the other two classes, $f$ must be trivial. Hence any element of $N_{G}(S)$ normalizes both $P$ and $R$. By (3.2) (ii) $N_{G}(S) \subseteq N_{G}(T)$. Hence $U=T P$ is a normal subgroup of $N_{G}(S)$. Similarly $V$ is normal. 
(3.4) The element $t=j_{2}$ of (2.2) in $S$ is not conjugate to any element of $Z$ in $G$.

Proof. Suppose that $t$ is conjugate to an element of $Z$ in $G$. Since $t$ is an element of $Z(T) \cap O$, the centralizer $W=C_{S}(t)$ is a subgroup of index $q$. Since $t$ is conjugate to an element of $Z$, there is a Sylow 2-group $S_{1}$ of $C_{G}(t)$ which contains $W . S_{1}$ is a Sylow 2-group of $G$. By (3.2), $S \cap S_{1}=W$. Suppose $q>2$. We apply the Proposition (4.5) of [32, IV] to $N_{G}(T) / T$. All assumptions of $(4.5)$ of [32, IV] are satisfied by (3.2) (iv) and (3.3). If $q>2,(4.5)$ of [32, IV] and (3.2) (iv) yield that $S \cap S_{1}=U$ or $V$. This is not the case.

If $q=2$, we use a different argument. Since $W$ is maximal, $S$ and $S_{1}$ are conjugate in $N_{G}(W)$. The center of $S_{1}$ is generated by $t$, so $t$ is conjugate to the unique involution $z$ of $N_{G}(W)$. We determine the upper central series of $W . W$ is the subgroup of $S$ consisting of the matrices $\left(a_{i j}\right)$ with $a_{21}=a_{n n-1}\left(\right.$ and $a_{i i}=1, a_{i j}=0$ for $\left.i<j\right)$. So $Z(W)$ $=\langle z, t\rangle$, and the second center $Z_{2}(W)$ coincides with $Z(T)$. Let $Z_{k}(W)$ be the $k$ th term in the upper central series of $W$. Then for $k \geqq 3$, $Z_{k}(W)$ is the subset of $S$ consisting of all $\left(a_{i j}\right)$ with $a_{i j}=0$ for $0<i-j$ $<n-k$. Let $l$ be the smallest integer such that $Z_{l}(W)$ is not of exponent 2. Thus $l=(n / 2)+1$ if $n$ is even, and $l=(n+1) / 2$ otherwise. The equation $X^{2}=z$ has more solutions in $Z_{l}(W)$ than the equation $X^{2}=t$. Hence $t$ is not conjugate to $z$ in $N_{G}(W)$. This contradicts the assumption.

(3.5) Suppose that an element of $Z$ is conjugate in $G$ to an element of $P-Z$ and that an element of $Z$ is conjugate to an element of $R-Z$ in $G$ also. Then $N_{G}(T) / T$ contains a normal subgroup of odd index which is a direct product of two groups isomorphic to $L_{2}(q)$.

Proof. By assumption there is an element $u$ of $P-Z$ which is conjugate to an element of $Z$. Since $P-Z$ is a conjugate class of $H$, we may assume that $u$ belongs to $Z(T)$. Then $C_{S}(u)=U$. So there is a Sylow 2-group $S_{1}$ of $G$ such that $S \cap S_{1}=U$. Similarly there is a Sylow 2-group $S_{2}$ of $G$ such that $S \cap S_{2}=V$.

Suppose that $q=2$. Then $Z$ is of order 2. Hence $N_{G}(S) \subseteq H$. By (2.7), $N_{H}(S)=S$, so we conclude $N_{G}(S)=S$. Therefore, $N_{G}(T) / T$ has an abelian Sylow 2-group which coincides with its normalizer. By a theorem of Burnside $N_{G}(T) / T$ has a normal 2-complement. Let $Q$ be the normal subgroup of index 4 such that $S \cap Q=T$. Then $Q / T$ is the normal 2-complement of $N_{G}(T) / T$. If $S_{1}$ is any Sylow 2-group of $N_{G}(T), Z\left(S_{1}\right) \subseteq Z(T)$ by (3.2) (i). If $z_{1}$ is any element of $Z(T)$ conjugate to $z, C_{G}\left(z_{1}\right) \cap N_{G}(T)$ is contained in a unique Sylow 2-group of $G$ by (2.11) (vi). Thus $N_{G}(T)$ contains at most 15 Sylow 2-groups. 
This yields $|Q / T| \leqq 15$. On the other hand $N_{G}(U)$ acts transitively on $Z(U)-1$. Hence $U / T$ commutes with precisely 3 elements of $Q / T$. Similarly $V / T$ commutes with 3 elements of $Q / T$. The Sylow group $S / T$ does not centralize any nonidentity element of $Q / T$. Hence $|Q / T|$ is divisible by 9 (the fixed point formula of Brauer [42]). Hence $|Q / T|=9$, and $Q / T$ must be elementary abelian. It is easy to check that $N_{G}(T) / T$ is a direct product of two groups isomorphic to $L_{2}(2)$.

We assume that $q>2$. All the assumptions of Proposition (4.6) of $\left[32\right.$, IV] are satisfied for $N_{G}(T) / T$ by (3.2) (iv), (3.3) and the assumptions. Hence by $(4.6)$ of $[32, \mathrm{IV}] N_{G}(T) / T$ contains a normal subgroup $L$ of odd index such that $L=L_{1} \times L_{2}$ and both $L_{1}$ and $L_{2}$ are isomorphic to $L_{2}(q)$.

(3.6) Under the same assumptions as (3.5), $Z(T)$ contains a conjugate subgroup $Z^{x}$ of $Z$ such that $Z^{x} \cap O=1$.

Proof. By (3.5), there is a Sylow 2-group $S_{1}$ of $N_{G}(T)$ such that $S \cap S_{1}=T$. Set $Z_{1}=Z\left(S_{1}\right)$. Since $Z_{1}$ centralizes $T, Z_{1} \subseteq Z(T)$. If $Z_{1} \cap O \neq 1$, there is an element $z_{1}$ of $Z_{1}$ such that $C_{S}\left(z_{1}\right)$ is of index $q$ in $S$. There is a Sylow 2-group $S_{2}$ of $C_{G}\left(z_{1}\right)$ which contains $C_{S}\left(z_{1}\right)$. Clearly $S_{2} \neq S_{1}$ and $S_{1} \cap S_{2}$ contains $T$. This is against (2.11) (vi). Thus $Z_{1} \cap O=1$.

Let $X$ be any subset of $H$, and let $z$ be an involution of $Z$. We say that $z$ fuses in $X$ if $z$ is conjugate to an element, $\neq z$, of $X$ in $G$.

We prove the following propositions.

(3.7) If $z$ does not fuse in $Z(T)-O$, then $z$ does not fuse in $H-O$.

(3.8) If $z$ does not fuse in $Z(T)-Z$, then $z$ does not fuse in $H-Z$.

Proposition (3.8) is an easy consequence of (3.7). Suppose that (3.7) holds. If $z$ does not fuse in $Z(T)-Z$, then $z$ does not fuse in $H-O$ by (3.7). But involutions of $O-Z$ are distributed into three conjugate classes of $H$ by $(2.11, \mathrm{v})$, each of which has a representative in $Z(T)$. Hence $z$ does not fuse in $O-Z$ either. This proves (3.8).

In proving (3.7) let us assume, by way of contradiction, $z$ fuses in $H-O$. A set of representatives from the conjugate classes of $H$ containing involutions of $H-O$ is given in (2.16). If $z$ is conjugate to the first element in (2.16), we say that a fusion of the first kind has occurred. Any other fusion of $z$ in $H$ is called of the second kind. We prove a series of lemmas under the assumption that $z$ does not fuse in $Z(T)-O$.

(a) No fusion of the first kind occurs. 
Proof. Assume that $z$ is fused to the first element of (2.16), which we denote by $u$. Since $u \notin Z(T)$ by assumption, we have $k \geqq 2$. Set

$$
Z_{1}=Z\left(C_{G}(u)\right) \text { and } Q=Z\left(C_{S}(u)\right) .
$$

Then $Z_{1}$ is a conjugate subgroup of $Z$. By (2.17) (i), $C_{S}(u)$ is a Sylow 2-group of $C_{G}(z, u)$. Since $Z_{1}$ is contained in $C_{G}(z, u)$, we have $Z_{1} \subseteq Q$.

By (2.17) (ii), $Q$ is a subgroup of order $q^{5}$ which contains $Z(T)$. Since $Z_{1}$ is not contained in $Z(T)$, we have $Z(T) \cap Z_{1}=1$ by (3.1) (ii). Let $A$ be a subgroup of $Z(T)$ consisting of matrices whose lower left corner is

$$
\left(\begin{array}{ll}
\alpha & \beta \\
0 & \delta
\end{array}\right) \quad \alpha, \beta, \delta \in F
$$

An easy computation shows that any element of the coset $A u$ is conjugate to $u$ in $S$.

Consider the fusion in $C_{G}(u)$. The element $u$ fuses to $z$. Since the center of a Sylow 2-group of $C_{G}(u, z)$ has order $q^{5}$, this fusion is of the first kind. Hence $Q$ contains $Z\left(T_{1}\right)$ for some conjugate subgroup $T_{1}$ of $T$. Moreover $Z\left(T_{1}\right)$ contains $Z_{1}$. Since $\left|Z\left(T_{1}\right)\right|=q^{4}$ and $|A|=q^{3}$, we have $\left|Z\left(T_{1}\right) \cap A\right| \geqq q^{2}$. The element $u$ is contained in $Z\left(T_{1}\right)$, so $Z\left(T_{1}\right)$ contains at least $q^{2}$ elements of the coset $A u$. Therefore $Z\left(T_{1}\right)$ contains at least $q^{2}$ elements which are conjugate to $z$. The same holds for $Z(T)$. By assumption no conjugate element of $z$ lies in $Z(T)-O$. Any element of $O$ which is conjugate to $z$ is contained in either $P$ or $R$ by (3.4). Since $P \cap Z(T)$ contains at most $q^{2}-1$ conjugate elements of $z, z$ fuses in both $P-Z$ and $R-Z$. By (3.6), $z$ fuses in $Z(T)-O$. This contradiction proves (a).

(b) Elements of $Z-1$ are conjugate to each other.

Proof. By assumption $z$ fuses to an element $u$ of $H-O$. By (a) we may assume that $u$ is one of the last three elements of (2.16). Set $Z_{1}=Z\left(C_{G}(u)\right)$ and $Q=Z\left(C_{S}(u)\right)$ as before. Then $Z_{1} \subseteq Q$ and $Z_{1} \cap Z(T)$ $=1$. A simple computation proves that elements of $Q-Z(T)$ are conjugate to each other in $N_{H}(S)$. This proves that elements of $Z_{1}-1$ are conjugate to $z$. The assertion (b) follows.

(c) If an element $z$ of $Z$ is conjugate to an element $u$ of $H-\langle z\rangle$, then $z$ is conjugate to the product $z u$.

Proof. If $u \in Z$, (c) follows from (b). If $u \in O-Z$, then $u$ belongs to either $P$ or $R$. Then the product $z u$ also belongs to $P-Z$ or $R-Z$ and is conjugate to $z$. If $u \in H-O$, we may assume that $u$ is one of the last three elements of (2.16). In all cases $z u$ is conjugate to $u$ in $S$. 
We can finish the proof of (3.7) as follows. Let $t$ be the element represented by $j_{2}$ of (2.2). Then by (3.4), $t$ is not conjugate to $z$ in $G$. By assumption $z$ is conjugate to one of the last three elements of (2.16), which is denoted by $u$. Then $k$ in (2.16) is larger than 1 for the second or third element, and is larger than 2 in the last case. Thus the element $u$ commutes with $t$ and is conjugate to $u t$ in $S$. Lemma (c) applied to $u$ yields that $u$ is conjugate to $u(u t)=t$. This is not the case.

4. Nonsimple cases. It is convenient to call an involution central if it is conjugate in $G$ to an involution of $Z$.

(4.1) If $Z(T)-Z$ contains no central involution, then either $Z$ is a normal subgroup of $G$, or $q=2$ and $H$ has a normal complement which is an abelian group of odd order.

Proof. By (3.8) there is no central involution in $H-Z$. Suppose that $q=2$. Then the involution $z$ of $Z$ does not fuse in $S$. By a theorem of Glauberman [13], $G$ is not simple. In fact, if $N$ is the largest normal subgroup of odd order of $G$, the coset $N z$ lies in the center of $G / N$. By Sylow's theorem, $G=N H$. Since $H$ contains no normal subgroup of odd order $>1$, we have $N \cap H=1$. Thus $N$ is a normal complement of $H$. $N$ is abelian because $z$ acts fixed point free.

Assume that $q>2$, and we prove a series of lemmas.

(a) Let $x$ be an element of $Z(T)-O$. Then $T$ is a Sylow 2-group of $C_{G}(x)$.

Proof. Choose a Sylow 2-group $T_{1}$ of $C_{G}(x)$ which contains $T$, and then choose a Sylow 2-group $S_{1}$ of $G$ which contains $T_{1}$. Since $T \subseteq S_{1}, Z\left(S_{1}\right)$ centralizes $T$ and hence is contained in $Z(T)$. By assumption $Z\left(S_{1}\right)=Z$. Then $S_{1}$ contains $O$. This $S=T O \subseteq S_{1}$. Since $C_{S}(x)=T$,

$$
T_{1} \subseteq C_{G}(x) \cap S=C_{S}(x)=T .
$$

This proves that $T$ is a Sylow 2-group of $C_{G}(x)$.

(b) Let $x$ be an element of $Z(T)-O$. Then any 2-element of $C_{G}(x)$ belongs to $H$.

Proof. Suppose false. Then there are Sylow 2-groups of $C_{G}(x)$ which are not contained in $H$. Choose a Sylow 2-group $Q$ of $C_{G}(x)$ such that $Q$ is not contained in $H$ and with this restriction $|Q \cap H|$ is maximal. Set $I=Q \cap H$. By definition $I$ contains the element $x$. Let $S_{1}$ be a Sylow 2-group of $G$ which contains $Q$. By definition $S_{1}$ is not contained in $H$. 
First we prove that $Z \cap I=1$. Suppose that $Z \cap I \neq 1$. Then $Z \cap I$ contains a central involution $z$. By assumption all central involutions of $S_{1}$ are contained in $Z\left(S_{1}\right)$. Hence we have $S_{1} \subseteq C_{G}(z)=H$, which is not the case.

Set $J=I C_{G}(I)$. We prove that $J / I$ is a $(T I)$-group. Since $Z$ centralizes $I$, there is a Sylow 2-group $J_{1}$ of $J$ which contains $Z I$. By definition $J \subseteq C_{G}(x)$. Let $T_{1}$ be a Sylow 2-group of $C_{G}(x)$ which contains $J_{1}$. Then $T_{1}$ contains $Z I$. By maximality, $T_{1} \subseteq H$. In particular $J_{1} \subseteq H$. Again by definition $Z\left(S_{1}\right)$ centralizes $I$. Let $J_{0}$ be a Sylow 2group of $J$ which contains $I Z\left(S_{1}\right)$, and let $T_{0}$ be a Sylow 2-group of $C_{G}(x)$ containing $J_{0}$. By definition $T_{0}$ contains $I$. If $T_{0}$ is contained in $H$, then $H$ contains $Z\left(S_{1}\right)$. By assumption this would imply $Z\left(S_{1}\right)=Z$ and $S_{1} \subseteq H$. Hence $T_{0}$ is not contained in $H$. The maximality of $|I|$ yields that $H \cap T_{0}=I$. This implies that $J_{1} \cap J_{0}=I$. Thus the group $J / I$ has two Sylow 2-groups which intersect trivially. Since $q>2$, a Sylow 2-group of $J / I$ contains more than one involution.

Any Sylow 2-group of $J$ contains $I$. Suppose that $J_{2}$ is a Sylow 2-group of $J$ such that $J_{1} \cap J_{2} \neq I$. Let $T_{2}$ be a Sylow 2-group of $C_{G}(x)$ which contains $J_{2}$. Then $T_{2} \cap H$ contains $J_{1} \cap J_{2}$ which has larger order than $I$. By maximality, $T_{2} \subseteq H$. Hence $Z$ centralizes $T_{2}$. In particular $Z$ centralizes $J_{\mathbf{2}}$. This implies that $Z \subseteq J_{2}$. All the assumptions of (2.20) are satisfied for $J / I$. It follows that $J / I$ is a $(T I)$ group.

By the structure theorem for (TI)-groups [34], there is a subgroup $K$ of $J$ of odd order such that $K$ normalizes a Sylow 2-group $J_{1}$ of $J$ and acts transitively on the set of involutions of $J_{1} / I$. Since $K$ normalizes $J_{1}$ and $Z$ is weakly closed in $J_{1}, K$ normalizes $Z$. This implies that $Z I / I$ contains all the involutions of $J_{1} / I$. By definition $T_{1}$ is a Sylow 2-group of $C_{G}(x)$. Hence $T_{1}$ is conjugate to $T$. We have shown that $T_{1} \subseteq H$. Let $L$ be a Sylow 2-group of $H$ which contains $T_{1}$. Set $M=Z\left(T_{1}\right)$. Then $M$ centralizes $I$ and $J_{1}$. Hence $M \subseteq J_{1}$. Since $M$ is elementary abelian, and since $Z I / I$ contains all the involutions of $J_{1} / I$, we conclude that $M \subseteq Z I$. The group $K$ is a subgroup of odd order in $J=I C_{G}(I)$. Hence $K \subseteq C_{G}(I)$. This implies that $[M, K]$ $\subseteq[Z I, K] \subseteq Z$. By definition $L \subseteq H$. Hence $[M, K, L] \subseteq[Z, L]=1$. Since $L$ normalizes $M$, we have $[L, M, K] \subseteq Z$. By the three subgroups lemma [17], we obtain $[K, L, M] \subseteq Z$. The groups $L$ and $M$ are conjugate to $S$ and $Z(T)$ respectively in $H$. Since $K$ normalizes $Z, K$ normalizes $H$. Thus $[K, L]$ is a subgroup of $H$. By an easy computation we prove that any element $h$ of $H$ satisfying $[h, M] \subseteq Z$ centralizes $M$. Hence we have $[K, L, M]=1$. The three subgroups 
lemma yields that $[L, M, K]=1$. This is a contradiction since $Z \subseteq[L, M]$ and $[Z, K] \neq 1$.

(c) $Z$ is a normal subgroup of $G$.

Proof. Suppose that (c) is false, and let $Z_{1}$ be a conjugate subgroup of $Z$ different from $Z$. Let $x$ be an element of $Z(T)-O$ and let $y$ be a nonidentity element of $Z_{1}$. Since $x$ is not central, there exists an involution $w$ of $G$ such that $w$ commutes with both $x$ and $y$, and that either $w x$ or $w y$ is central ((2.18)). By (b), $w$ is contained in $H$, so is $w x$.

If $w x$ is central, $w x \in Z$ by assumption. Hence $w \in Z(T)-O$, and $y \in H$ by (b). Since $y$ is central, this implies that $y \in Z$. This is not the case as $Z \cap Z_{1}=1$.

If, on the other hand, wy is central, wy $\in Z_{1}$ because wy commutes with $y$. This implies that $w \in Z_{1}$; in particular, $w$ is central. Since $w \in H$, we have $w \in Z$. This is a contradiction because $w \in Z \cap Z_{1}$.

(4.2) Suppose that $P-Z$ contains a central involution. Then there exists precisely one conjugate class of central involutions.

Proof. Let $z_{1}$ be a central involution of $P-Z$. Set $Z_{1}=Z\left(C_{G}\left(z_{1}\right)\right)$. Since $P$ is self-centralizing, $Z_{1} \subseteq P$ by (3.1) (ii). We have $Z \cap Z_{1}=1$. By (2.11) (v) elements of $Z_{1}-1$ are conjugate to each other in $H$. This proves (4.2).

(4.3) Suppose that $P-Z$ contains a central involution, but no involution of $Z(T)-O$ is central. Then $P$ is a normal subgroup of $G$.

Proof. Propositions (3.4), (3.6) and (3.7) yield that no involution of $H-P$ is central. By assumption every involution of $P$ is central. Set

$$
O^{\prime}=Z(T) \cap O \text { and } \quad Y=Z(T) \cap P .
$$

We prove a series of lemmas.

(a) $N_{G}(P)$ acts transitively on the set of involutions of $P$.

Proof. The group $H$ is a subgroup of $N_{G}(P)$ and acts transitively on $P-Z$. Let $y$ be an element of $Y-Z$. Then $C_{S}(y)=T P=U$. Let $S_{1}$ be a Sylow 2-group of $C_{G}(y)$ which contains $U$. By (3.2) (iii), $S_{1} \subseteq N_{G}(T)$; in particular $S_{1}$ normalizes $U$. Since $S$ and $S_{1}$ are two Sylow subgroups of $N_{G}(U)$, there is an element $x$ of $N_{G}(U)$ such that $S_{1}=S^{x}$. Involutions of $P^{x}$ are central, and no central involution lies in $U-P$. Hence $P^{x}=P$. Thus $x$ is an element of $N_{G}(P)$. Since $P$ 
is self-centralizing, $Z^{x} \subseteq P$. Clearly $Z \cap Z^{x}=1$. If $x$ is an involution of $Z, z^{x}$ is an element of $P-Z$. Hence $z^{x}$ is conjugate to $y$ in $H$. Since $x \in N_{G}(P), z$ is conjugate to $y$ in $N_{G}(P)$. The lemma (a) follows immediately.

(b) $N_{G}(P)-P$ contains no central involution.

Proof. Since $P$ is a 2-group, any involution of $N_{G}(P)$ centralizes some involution of $P$. Let $u$ be a central involution of $N_{G}(P)$. There is an involution $v$ of $P$ which commutes with $u$. Let $z$ be an involution of $Z$. By (a), there is an element $x$ of $N_{G}(P)$ such that $z=v^{x}$. Then $u^{x}$ is a central involution of $C_{G}(z)=H$. This implies $u^{x} \in P$ and hence $u \in P$.

(c) $P$ is a TI-set: that is, $P \cap P^{x} \neq 1$ implies $P=P^{x}$.

Proof. Let $z$ be an involution of $Z$. Suppose that $P \cap P^{x} \neq 1$ and choose an involution $u$ of $P \cap P^{x}$. By (a), there is an element $v$ of $N_{G}\left(P^{x}\right)$ such that $z^{x v}=u$. Similarly there is an element $w$ of $N_{G}(P)$ satisfying $u=z^{w}$. The element $x v w^{-1}$ belongs to $H$. Since $P$ is a normal subgroup of $H$, we have $P^{w}=P^{x v}$. By definitions of $v$ and $w$,

$$
P=P^{w}=P^{x v}=P^{x} .
$$

(d) An element of $Z(T)-O$ is conjugate to an element of $O^{\prime}-Y$.

Proof. Let $N$ be the subgroup of $N_{G}(U)$ which is generated by Sylow 2-groups. By (3.2) (ii), $N$ normalizes $T$, so $N$ acts on $U / T$. Since $T$ contains the commutator subgroup of $S, N$ acts trivially on $U / T$. We have $Y=Z(U)$, so $N$ acts on $Y$ and $Z(T) / Y$. The subgroup $U$ acts trivially on $Y$ and $Z(T) / Y$. Set $U_{0}=N / U$. Then both $Y$ and $Z(T) / Y$ are $U_{0}$-groups. We claim that they are $U_{0}$-isomorphic. Choose an element $u$ of $U-T$. The mapping

$$
x \rightarrow[x, u] \quad(z \in Z(T))
$$

defines a homomorphism of $Z(T)$ into $Y$. The kernel of this homomorphism is $Z(T) \cap C_{G}(u)$. Since $u \in U-T$, we have $Z(T) \cap C_{G}(u)$ $=Y$. Thus the above homomorphism induces an isomorphism $f$ of $Z(T) / Y$ onto $Y$. We have $f(Y x)=[x, u]$. Let $v$ be an element of $N$. Then $v$ acts trivially on $U / T$. Hence $u^{v}=t u$ for some element $t$ of $T$. It follows that

$$
f\left(Y x^{v}\right)=\left[x^{v}, u\right]=\left[x^{v}, u^{v}\right]=f(Y x)^{v} .
$$

This proves that $f$ is a $U_{0}$-isomorphism of $Z(T) / Y$ onto $Y$. Every involution of $Y$ is contained in the center of some Sylow 2-group of $N_{G}(U)$ and hence conjugate to an involution of $Z$. This implies that 
every element of $Z(T) / Y$ is conjugate to an element of $f^{-1}(Z)$. By definition $f^{-1}(Z)=O^{\prime} / Y$. This yields the assertion.

(e) Let $x$ be an element of $O^{\prime}-Y$. Then every 2-element of $C_{G}(x)$ is contained in $N_{G}(P)$.

Proof. Let $y$ be an element of $Y-Z$ and let $z$ be an involution of $Z$. By assumption both $y$ and $z$ are central. We have $C_{G}(x, z)=C_{H}(x)$. By $(2.11, \mathrm{iv}), C_{S}(x)$ is a Sylow 2-group of $C_{H}(x)$ and $\left|S: C_{S}(x)\right|=q$. On the other hand, a Sylow 2-group of $C_{G}(x, z)$ has order equal to $|T|$. This implies that $y$ and $z$ are not conjugate in $C_{G}(x)$.

Set $W=C_{S}(x)$. Let $W_{1}$ be a Sylow 2-group of $C_{G}(z)$ which contains $W$. There is a Sylow 2-group $S_{1}$ of $G$ containing $W_{1}$. Then $Z\left(S_{1}\right)$ centralizes $Z$ and so normalizes $P$ by (c). This proves that $Z\left(S_{1}\right) \subseteq P$. Since $Z\left(S_{1}\right)$ centralizes $T$, it is contained in $Z(T)$. Therefore $Z\left(S_{1}\right)$ $\subseteq P \cap Z(T)=Y$. There exists a Sylow 2-group of $C_{G}\left(Z\left(S_{1}\right)\right)$ which contains $U=C_{S}\left(Z\left(S_{1}\right)\right)$. By uniqueness (2.11) (vi), $S_{1}$ contains $U$. Suppose that $S_{1} \neq S$. Then $U=S \cap S_{1}$ and $W=U$. Since $x \in Z(W)$, we have $x \in Z(U)=Y$, which is not the case. Hence $S_{1}=S$ and this implies that $W$ is a Sylow 2-group of $C_{G}(x)$.

Let $W^{\prime}$ be any Sylow 2-group of $C_{G}(x)$. Choose an element $z^{\prime}$ of $Z\left(W^{\prime}\right)$ which is conjugate to $z$ in $C_{G}(x)$. Then $z^{\prime}$ is not conjugate to $y$ in $C_{G}(x)$. By (2.18) there exists an involution $w$ of $C_{G}(x)$ such that $w$ commutes with both $y$ and $z^{\prime}$, and $w y$ is central. The element $w y$ commutes with an element $y \neq 1$ of $P$. By (c), wy normalizes $P$, and hence by (b) $w y \in P$. Thus $w \in P$. Since $z^{\prime}$ centralizes $w \in P$, the same argument proves $z^{\prime} \in P$. Therefore $C_{G}\left(z^{\prime}\right) \subseteq N_{G}(P)$. Since $W^{\prime} \subseteq C_{G}\left(z^{\prime}\right)$, we have $W^{\prime} \subseteq N_{G}(P)$.

(f) $P$ is a normal subgroup of $G$.

Proof. Suppose false and let $P^{\prime}$ be a subgroup, $\neq P$, conjugate to $P$. Let $x$ be an element of $O^{\prime}-Y$ and $z$ be an involution of $P^{\prime}$. Then $x$ is not conjugate to $z$ in $G$. There exists an involution $w$ of $C_{G}(x, z)$ such that $x w$ is conjugate to $x$ or $z$.

Suppose that $x w$ is conjugate to $x$. Then $w z$ is conjugate to $z$. This implies that $w z \in P^{\prime}$ and $w \in P^{\prime}$. On the other hand $w \in N_{G}(P)$ by (e). Thus $w$ is a central involution of $N_{G}(P)$. It follows from (b) that $w \in P$. This is a contradiction because $P \cap P^{\prime}=1$ by (c).

Suppose that $x w$ is conjugate to $z$. Then $x w$ is a central involution which lies in $N_{G}(P)$. Hence by (b), $x w \in P$. Since $x \in O^{\prime}-Y$ and $P \subseteq O$, we conclude that $w \in O-P$. The element $w$ is conjugate to an element of $O^{\prime}-Y$ in $N_{G}(P)$. By (e), any 2-element of $C_{G}(w)$ is contained in $N_{G}(P)$. This implies that $z$ is a central involution of $N_{G}(P)$. By (b), we have $z \in P$, which is a contradiction. 
5. Structure of some local subgroups. In this section we make the following standing assumption:

$P-Z$ contains a central involution.

This assumption implies that there is precisely one conjugate class of central involutions by (4.2).

We choose a complement $K_{0}$ of $S$ in $N_{H}(S)$ and fix it throughout discussion. The structure of $K_{0}$ was stated in (2.7). $K_{0}$ is isomorphic to the factor group of the group of $(n-2)$-tuples $\left(\mu_{1}, \cdots, \mu_{n-2}\right)$ of nonzero elements $\mu_{1}, \cdots, \mu_{n-2}$ of $F$ (equipped with the componentwise multiplication) by the subgroup consisting of $(\lambda, \cdots, \lambda)$ where $\lambda^{n}=1$. Thus $K_{0}$ is an abelian group of exponent $q-1$ and order $(q-1)^{n-2} / d$ where $d$ is the greatest common divisor of $n$ and $q-1$.

There is a complement of $S$ in $N_{G}(S)$ which contains $K_{0}$. Let $K$ be such a complement. We will fix $K$ too. If $q=2, N_{G}(S)$ centralizes $Z$, so we have $K=1$.

(5.1) If $q>2, C_{S}(K)=1$.

Proof. Clearly we have $C_{S}(K) \subseteq C_{S}\left(K_{0}\right)$. By $(2.7), C_{S}\left(K_{0}\right)=Z$. The assumption at the beginning of this section implies that involutions of $Z$ are conjugate. By Burnside's lemma [9], they are conjugate in $N_{G}(S)$. Therefore $K$ acts transitively on the involutions of $Z$; in particular $K$ does not centralize $Z$ if $q>2$.

The strucutre of $K$ emerges from the study of the structure of $N_{G}(U)$.

(5.2) $N_{G}(U)$ contains a normal subgroup $L$ of odd index such that $L / U \cong L_{2}(q)$. Furthermore, $L$ contains an involution $t$ such that

$$
N_{G}(U)=\langle S, K, t\rangle \text { and } t \in N_{G}(K) \text {. }
$$

Proof. By assumption there is a Sylow 2-group $S_{1}$ of $N_{G}(U)$ such that $S \cap S_{1}=U$ (the first paragraph of the proof of (3.5)). Since $U$ is a maximal intersection of Sylow groups, $N_{G}(U) / U$ is a $(T I)$-group.

If $q=2$, we set $L=N_{G}(U)$ and verify the assertions easily. So assume that $q>2$. The group $N_{G}(U) / U$ is a $(T I)$-group with an abelian Sylow 2-group of order $q$. Hence by the structure theorem of (TI)-groups [34], $N_{G}(U)$ contains a normal subgroup $L$ of odd index such that $L / U \cong L_{2}(q)$. Since $L$ is normal, we have

$$
N_{G}(U)=L N_{G}(S)=L K .
$$

By (4.4) of $[32, \mathrm{IV}], N_{G}(U)$ contains an element $x$ such that $x^{2} \in U$ and $x$ normalizes $U K$. Set $X=\langle U K, x\rangle$. Then $X=U N_{X}(K)$. Since $U$ is normal, $N_{X}(K) \cap U$ centralizes $K$. By (5.1) we have $C_{S}(K)=1$. 
This implies that $N_{X}(K) \cap U=1$ and the extension of $X$ over $U$ splits. Hence there is an involution $t$ in the coset $U x$ such that $t \in N_{X}(K)$ and $\langle S, t\rangle=L$. It follows that $N_{G}(U)=\langle S, K, t\rangle$.

(5.3) $K$ is isomorphic to the direct product of $K_{0}$ and the cyclic group of order $q-1$.

Proof. We may assume that $q>2$. Let $L$ be the normal subgroup of $N_{G}(U)$ given in (5.2). Since $L / U$ is isomorphic to $L_{2}(q), L \cap K$ is a cyclic group of order $q-1$. The factor group $L / U$ acts on $Z(U)$ and the action is the natural one. Hence $L \cap K$ acts regularly on $Z . K$ induces a permutation group on $Z-1$. By assumption (2), the stabilizer of an involution of $Z$ stabilizes all elements of $Z$. Thus $K_{0}$ is the stabilizer and is a normal subgroup of index $q-1$. Since $L \cap K_{0}=1$, we have $K=K_{0}(L \cap K)$. Since $L$ is normal, $L \cap K$ is a normal subgroup of $K$. Thus, $K=K_{0} \times(L \cap K)$.

(5.4) Suppose that $N_{G}(Z)$ does not contain $N_{G}(P)$. Then $N_{G}(P)$ has a series of normal subgroups $1 \subset P \subset G_{1} \subset G_{2} \subset N_{G}(P)$ such that $G_{1} / P$ is a cyclic group of order $(q-1) / d$,

$$
G_{2} / G_{1} \cong L_{n-1}(q) \text { and } N_{G}(P) / G_{1} \cong P G L(n-1, q) .
$$

Furthermore $\left[N_{G}(P), G_{1}\right] \subseteq P$.

Proof. As in (4.3, a), $N_{G}(P)$ acts transitively on the involutions of $P$. Let $Q$ be the subgroup of $S$ consisting of matrices of the form

$$
\left(\begin{array}{ll}
I & \\
X & I
\end{array}\right)
$$

where $X$ is an $(n-2,2)$ matrix. Then $Q$ is an elementary abelian subgroup and self-centralizing. The intersection $P \cap Q$ is of order $q^{n-2}$. We define an incidence structure $P=(\mathfrak{B}, \mathfrak{B}, I)$ as follows:

$\mathfrak{B}$ : the totality of conjugates of $Z$,

$\mathfrak{B}:$ the totality of conjugates of $P \cap Q$,

$I$ : containment.

Elements of $\mathfrak{B}$ are called points and elements of $\mathfrak{B}$ are blocks. $N_{G}(P)$ induces a group of automorphisms of this structure. By assumption $N_{G}(P)$ induces a doubly transitive permutation group on $\mathfrak{B}$. We want to prove that $N_{G}(P)$ is doubly transitive on $\mathfrak{B}$. This follows if $P$ is a symmetric design by a theorem of Parker [29]. We use the same idea. Let $I$ be the incidence matrix of $P$. If $X$ is a point, the number of blocks containing $X$ is a constant, say $a$, since $N_{G}(P)$ is transitive on $\mathfrak{B}$. Similarly the number of blocks incident with two 
distinct points is another constant, say $b$. Since a block can not contain all points, we have $a \neq b$. Clearly $a \geqq 1$. If ${ }^{t} I$ is the transposed matrix of $I$, then the entries off the main diagonal of $I^{t} I$ are $b$; while the diagonal entries are $a$. Hence $I^{t} I$ is nonsingular. This implies that $|\mathfrak{B}| \leqq|\mathfrak{B}|$. If $|\mathfrak{P}|=|\mathfrak{B}|, I$ is nonsingular and intertwines the representations on points and blocks. So $N_{G}(P)$ is doubly transitive on $\mathfrak{B}$.

We prove that points on a block form a partiton of involutions of a block (remember that a block is a subgroup). Let $X$ be a point and $Y$ be a block. Suppose that $X \cap Y$ contains an involution $x$. The point $X$ is a conjugate of $Z$ and $Y=P \cap Q^{\nu}$ for some $y \in N_{G}(P)$. Both $P$ and $Q^{y}$ are self-centralizing abelian subgroups. Hence by (3.1), $X$ is contained in both $P$ and $Q^{\nu}$. Thus, $X \cap Y \neq 1$ implies $X \subseteq Y$. Since every involution of $P$ is contained in some conjugate subgroup of $Z$, a block is partitioned.

We will prove $|\mathfrak{B}|=|\mathfrak{B}|$. We have

$$
|\mathfrak{B}|=\left(q^{n-1}-1\right) /(q-1) .
$$

Suppose that $|\mathfrak{P}| \neq|\mathfrak{B}|$. Since $|\mathfrak{B}| \leqq|\mathfrak{B}|$, we have $|\mathfrak{P}|<|\mathfrak{B}|$. Hence there is a pair $(X, Y)$ of blocks $X$ and $Y$ such that $X \neq Y$ and $X Y$ is a proper subgroup of $P$. In particular $|X Y: X|<q$. Since the block $Y$ is partitioned into points, there is a point $W$ in $Y$ which is not contained in $X$. Then by (3.1) we have $X \cap W=1$. Hence

$$
q=|X W: X| \leqq|X Y: X|<q,
$$

a contradiction.

We have shown that $N_{G}(P)$ induces a doubly transitive permutation group on $\mathfrak{B}$. The stabilizer of the point $Z$ is $N_{G}(Z)$, and clearly it is transitive on blocks incident with $Z$. It is easy to see that $Z$ is the intersection of all the blocks which contain $Z$. Let $G_{1}$ be the kernel of the representation of $N_{G}(P)$ on $\mathfrak{B}$. Then $G_{1}$ stabilizes all the points, and coincides with the kernel of the representation of $N_{G}(P)$ as a permutation group on $\mathfrak{B}$. We have $H \cap G_{1}=P$, as the elements of $H \cap G_{1}$ centralizes $P$. We apply a theorem of Ito [21] to $N_{G}(P) / G_{1}$. The group $O G_{1} / G_{1}$ leaves all the blocks incident with $Z$ invariant. We conclude that $N_{G}(P) / G_{1}$ is isomorphic to a subgroup of the group of all the projectivities of the projective space of dimension $n-2$ over $F$, and that $N_{G}(P) / G_{1}$ contains a normal subgroup $G_{2} / G_{1}$ which is isomorphic to $L_{n-1}(q)$. If $q=2$, the order of $N_{G}(P) / P$ is equal to that of $L_{n-1}(2)$. Hence we have $P=G_{1}$. This proves (5.4) for $q=2$.

It remains to prove that $N_{G}(P) / G_{1} \cong \operatorname{PGL}(n-1, q)$ and $G_{1} / P$ is the cyclic group of order $(q-1) / d$ when $q>2$. Consider the action of 
$K$ on $P$. Since $|K|$ is relatively prime to $|P|, P$ is completely reducible as $K$-module. Since $K$ is abelian of exponent $q-1$ by (5.3), each irreducible $K$-invariant subgroup of $P$ is of order $q$ (recall that $C_{P}(K)=1$ by $\left.(5.1)\right)$. Hence we have

$$
P=Z+P_{1}+\cdots+P_{n-2},
$$

where each $P_{i}$ is an irreducible $K$-invariant subgroup of order $q$. $K$ acts on the set of involutions of $P_{i}$ transitively. Since $K$ is abelian, the index of $C_{K}\left(P_{i}\right)$ in $K$ is $q-1$. By (5.3) we have $|K|=(q-1)^{n-1} / d$. Let $K_{1}$ be the intersection of all $C_{K}\left(P_{i}\right), i=1, \cdots, n-2$. Then $K_{1}$ acts semiregularly on the set of involutions of $Z$, and we have $\left|K_{1}\right| \geqq(q-1) / d$. Since $K_{1} \cap K_{0}=1, K_{1}$ is by (5.3) a cyclic group whose order divides $q-1$.

By (2.7), elements of $K_{0}$ correspond to matrices with $\mu_{1}, \cdots, \mu_{n-2}$ on the main diagonal. There are irreducible $K_{0}$-invariant subgroups $Q_{i}$ of $P$ on which $K_{0}$ acts as the scalar multiplication of the field element $\mu^{-1} \mu_{i}$ for $i=1,2, \cdots, n-2$, where $\mu^{2} \mu_{1} \cdots \mu_{n-2}=1$. The groups $Q_{1}, \cdots, Q_{n-2}$ are mutually nonisomorphic as $K_{0}$-modules. Hence they are the only irreducible $K_{0}$-invariant subgroups of $P$ not contained in $Z$. This proves that the set $\left\{Q_{1}, \cdots, Q_{n-2}\right\}$ coincides with the set $\left\{P_{1}, \cdots, P_{n-2}\right\}$. Let $\omega$ be a generator of the multiplicative group $F^{*}$ of $F$. If $x$ is an element of $K_{0}$ corresponding to the matrix for which

$$
\mu_{1}=\mu_{2}=\cdots=\mu_{n-2}=\omega^{2},
$$

$x$ acts on each $P_{i}$ as the scalar multiplication of $\omega^{n}$. As shown before the subgroup $K_{1}$ acts on $Z$ as a cyclic subgroup of $F^{*}$ of order at least $(q-1) / d$. By definition, $d$ is the greatest common divisor of $q-1$ and $n$. Hence $K_{1}$ contains an element $y$ which acts as the multiplication of $\omega^{n}$. The product $x y$ acts as the multiplication of $\omega^{n}$ on $P$ considered as a vector space over $F$, so that $x y \in G_{1}$. This proves that $G_{1} / P$ contains a cyclic subgroup of order $(q-1) / d$.

The incidence structure $P$ defined earlier is the block design determined by the one-dimensional and $(n-2)$-dimensional subspaces of the vector space $P$ over $F$. Each element of $H$ induces a linear projectivity on $P$. In fact, if an element $h$ of $H$ corresponds to the matrix

$$
\left(\begin{array}{lll}
x & & \\
& Y & \\
& & x
\end{array}\right), \quad x^{2}(\operatorname{det} Y)=1,
$$

then $h$ transforms an element of $P$ 


$$
\left(\begin{array}{lll}
1 & & \\
A & I & \\
z & & 1
\end{array}\right) \text { into }\left(\begin{array}{ccc}
1 & & \\
x Y^{-1} A & I & \\
z & & 1
\end{array}\right)
$$

We have

$$
\operatorname{det}\left(x Y^{-1}\right)=x^{n-2}(\operatorname{det} Y)^{-1}=x^{n} .
$$

The index of $\operatorname{PSL}(n-1, q)$ in $\operatorname{PGL}(n-1, q)$ is the greatest common divisor of $n-1$ and $q-1$. Hence the image of $H$ in $N_{G}(P) / G_{1}$ is a subgroup of $\operatorname{PGL}(n-1, q)$ and covers the section

$$
\operatorname{PGL}(n-1, q) / \operatorname{PSL}(n-1, q) \text {. }
$$

Thus, $N_{G}(P) / G_{1}$ contains a subgroup isomorphic to $\operatorname{PGL}(n-1, q)$. We have

$$
\begin{aligned}
\left|N_{G}(P)\right| & =\left(q^{n-1}-1\right)|H|=|P||\mathrm{GL}(n-1, q)| / d \\
& =|P||\operatorname{PGL}(n-1, q)|(q-1) / d .
\end{aligned}
$$

On the other hand,

$$
\begin{gathered}
\left|N_{G}(P): P\right|=\left|N_{G}(P): G_{1}\right|\left|G_{1}: P\right| \\
(q-1) / d \leqq\left|G_{1}: P\right| \text { and }|\operatorname{PGL}(n-1, q)| \leqq\left|N_{G}(P): G_{1}\right| .
\end{gathered}
$$

Hence we have the equality signs in both places. This implies that

$$
N_{G}(P) / G_{1} \cong \mathrm{PGL}(n-1, q)
$$

and $G_{1} / P$ is the cyclic group of order $(q-1) / d$. Since $L_{n-1}(q)$ is simple, we conclude that $N_{G}(P)$ is generated by conjugates of $H$. This yields that $\left[N_{G}(P), G_{1}\right] \subseteq P$.

(5.5) $N_{G}(Z)$ does not contain $N_{G}(P)$, unless $n=5$ and $q=2$.

Proof. Suppose that $N_{G}(P) \subseteq N_{G}(Z)$. By (5.2), $N_{G}(U)$ contains an involution $t$ such that $S_{1}=S^{t}$ is a Sylow 2-group of $N_{G}(U)$ different from $S$. Set $Z_{1}=Z\left(S_{1}\right)$. Then $Z_{1}$ is contained in $Z(U)$. By definition (2.9), $U=T P$. Hence we have

$$
Z(U)=P \cap Z(T) \subseteq P
$$

Thus $Z(U)=Z \times Z_{1}$ and $t$ exchanges $Z$ and $Z_{1}$. Since $N_{G}(P) \subseteq N_{G}(Z)$, $P^{t}$ is different from $P$. The element $t$ normalizes $U$ and hence $T$ by (3.2) (ii). This proves that $P^{t}$ is an elementary abelian subgroup of $U$, which is normalized by $T$ but not contained in $T$. The structure of such a group has been studied in (2.12) (i). 
Since $P$ is normalized by $C_{G}(Z), P^{t}$ is normalized by $C_{G}\left(Z_{1}\right)$, in particular by $C_{H}\left(Z_{1}\right)$. We verify easily that the element corresponding to the matrix

$$
\left(\begin{array}{lll}
x & & \\
& Y & \\
& & x I_{2}
\end{array}\right) \quad\left(x^{3}(\operatorname{det} Y)=1\right)
$$

belongs to $C_{H}\left(Z_{1}\right)$. By (2.12) (i), elements of $P^{t}$ are of the form

$$
\left(\begin{array}{lll}
1 & & \\
A & I & \\
C & D & I
\end{array}\right)
$$

where $D$ is a $2 \times(n-2)$ matrix such that the columns, except possibly the first and the second, are zero. Since $C_{H}\left(Z_{1}\right)$ normalizes $P^{t}$, the matrix

$$
\left(\begin{array}{lll}
x & & \\
& Y & \\
& & x I
\end{array}\right)^{-1}\left(\begin{array}{lll}
1 & & \\
A & I & \\
C & D & I
\end{array}\right)\left(\begin{array}{lll}
x & & \\
& Y & \\
& & x I
\end{array}\right)=\left(\begin{array}{lll}
1 & & \\
A^{\prime} & I & \\
C^{\prime} & D^{\prime} & I
\end{array}\right)
$$

belongs to $P^{t}$ for any $x$ and $Y$ such that $x^{3}(\operatorname{det} Y)=1$. Taking det $Y$ $=x=1$, we compute (5.6) and find that $D^{\prime}=D Y$. If $n \geqq 6$, it is obvious that $D^{\prime}$ does not have the form $(E, O)$ with a $2 \times 2$ submatrix $E$ for some choice of $Y$ with determinant 1 . This yields that $n=5$.

By (2.12) (i), the submatrix $D$ is determined by $A$; in fact for some $u$ and $v$

$$
D=\left(\begin{array}{cc}
\beta u & \alpha u \\
\beta v & \alpha v
\end{array}\right) \quad \text { if } A=\left(\begin{array}{l}
\alpha \\
\beta
\end{array}\right) .
$$

Similarly $D^{\prime}$ is determined by $A^{\prime}$ with the same $u$ and $v$. Set

$$
Y=\left(\begin{array}{ll}
\lambda & \mu \\
\nu & \eta
\end{array}\right) \text { and } \Delta=\operatorname{det} Y .
$$

Then we have

$$
Y^{-1}=\frac{1}{\Delta}\left(\begin{array}{ll}
\eta & \mu \\
\nu & \lambda
\end{array}\right)
$$

By (5.6), $x Y^{-1} A=A^{\prime}$ and $x^{-1} D Y=D^{\prime}$. This yields that

$$
\beta^{\prime}=x^{-1}(\beta \lambda+\alpha \nu)=(x / \Delta)(\nu \alpha+\lambda \beta) .
$$


Note that either $u$ or $v$ is nonzero as $P \neq P^{t}$. Since (5.7) holds for any $\alpha$ and $\beta$, we must have $x^{-1}=x / \Delta$, or $\Delta=x^{2}$. The value $\Delta$ of the determinant is arbitrary except that there must be an $x$ such that $x^{3} \Delta=1$. Since $\Delta=x^{2}$, we have $\Delta^{5}=1$. Since $\Delta$ may be an arbitrary third power, we have $\omega^{15}=1$ for all nonzero elements of $F$. Thus $q=2,4$ or 16 . But if $q-1$ is divisible by 3 , we can choose $x \neq 1$ such that $x^{3}=1$. Then for $\Delta=1$, we have $\Delta=x^{2}=1$. This is a contradiction. Hence we have $q=2$.

(5.8) $P$ is a (TI)-set and $N_{G}(P)$ acts transitively on the set of involutions, unless $n=5$ and $q=2$.

Proof. The second assertion follows easily from (5.5). Proof same as the one of (4.3) (c) yields the first.

If we make the assumption:

$$
R-Z \text { contains a central involution, }
$$

then the argument of this section yields propositions similar to (5.2) and (5.4) which determine the structure of $N_{G}(V)$ and $N_{G}(R)$ respectively.

(5.9) Suppose that $R-Z$ contains a central involution. Then $N_{G}(V)$ contains a normal subgroup of odd index, which is isomorphic to $L_{2}(q)$. Furthermore there is an involution s such that

$$
N_{G}(V)=\langle S, K, s\rangle \text { and } s \in N_{G}(K) \text {. }
$$

(5.10) Suppose that $N_{G}(R)$ is not a subgroup of $N_{G}(Z)$. Then $N_{G}(R)$ contains a normal subgroup $G_{3}$ such that $P \subseteq G_{3} \subseteq N_{G}(R), G_{3} / P$ is the cyclic group of order $(q-1) / d, N_{G}(R) / G_{3}$ is isomorphic to $\operatorname{PGL}(n-1, q)$ and $\left[N_{G}(P), G_{3}\right] \subseteq P$.

(5.11) $N_{G}(R)$ is not a subgroup of $N_{G}(Z)$ unless $n=5$ and $q=2$.

Propositions (5.5) and (5.11) may be stated in a slightly different manner.

(5.12) We have $N_{G}(U) \subseteq N_{G}(P)$ and $N_{G}(V) \subseteq N_{G}(R)$, unless $n=5$ and $q=2$.

Proof. Suppose that either $n \geqq 5$, or $q>2$. The proof of (5.5) actually shows that an element $t$ of $N_{G}(U)-N_{G}(S)$ belongs to $N_{G}(P)$. It follows from (5.2) that

$$
N_{G}(U)=\langle S, K, t\rangle \subseteq N_{G}(P) .
$$

Similarly we prove $N_{G}(V) \subseteq N_{G}(R)$ 
6. Construction of a subgroup isomorphic to $L_{n}(q)$. In this section we assume

(6.1) (i) $P-Z$ contains a central involution;

(ii) $Z(T)-O$ contains a central involution;

(iii) either $n \geqq 6$ or $n=5$ and $q>2$.

All propositions of this section are proved under the set of assumptions (6.1). Our goal is to construct a subgroup $G_{0}$ which is isomorphic to $L_{n}(q)$. Simple consequences of (6.1) are the following.

(6.2) (i) There is precisely one conjugate class of central involutions.

(ii) $N_{G}(U)$ involves $L_{2}(q)$; in particular there exists a Sylow 2-group $S_{1}$ such that $S \cap S_{1}=U$.

(iii) $N_{G}(P)$ involves $L_{n-1}(q)$, and has the structure stated in (5.4).

Proof. Proposition (i) is the restatement of (4.2), and (ii) follows from (5.2). The last proposition (iii) is a consequence of (5.4), (5.5) and the assumption (6.1) (iii).

(6.3) (i) $R-Z$ contains a central involution.

(ii) $N_{G}(V)$ involves $L_{2}(q)$, and has the similar structure as $N_{G}(U)$.

(iii) $N_{G}(R)$ contains a normal subgroup $G_{3}$ such that $G_{3} / R$ is the cyclic group of order $(q-1) / d$ and $N_{G}(R) / G_{3} \cong \mathrm{PGL}(n-1, q)$.

(iv) $N_{G}(T) / T$ contains a normal subgroup of odd index which is a direct product of two copies of $L_{2}(q)$.

Proof. By assumption (6.1) (ii) there is a central involution $z$ in $Z(T)-O$. We have $C_{S}(z)=T$ by (2.11) (iv). Let $S_{0}$ be a Sylow 2-group of $C_{G}(z)$ which contains $T$. Since $z$ is central, $S_{0}$ is a Sylow 2-group of $G$. We have $S \cap S_{0}=T$. We apply (2.19) to $N_{G}(T) / T$. By (6.2) (ii), there is a Sylow 2-group $S_{1}$ such that $S \cap S_{1}=U$. Hence by (2.19) there must be a Sylow 2-group $S_{2}$ of $N_{G}(T)$ such that $S \cap S_{2} \neq T$ and $S \cap S_{2}$ does not contain $U$. Set $W=S \cap S_{2}$.

Suppose that $q=2$. Then there are precisely three maximal subgroups of $S$ containing $T$. Since $S_{2} \neq S$, we have $Z\left(S_{2}\right) \neq Z$ by (2.11) (vi). Hence the central involution of $Z\left(S_{2}\right)$ is contained in $(Z(T) \cap O)$ $-Z$. Since $W \neq U$, we have $W=V$ and the central involution of $Z\left(S_{2}\right)$ lies in $R-Z$. This proves (i) for $q=2$. Suppose that $q>2$. By (4.5) of $\left[32\right.$, IV] and (3.3), we have $W=V$. This implies that $Z\left(S_{2}\right) \subseteq R$ and $Z\left(S_{2}\right) \neq Z$. Thus (i) is proved.

Propositions (ii) and (iii) are proved in the same way as (6.2) (ii) and (6.2) (iii). The last assertion follows from (3.5), (6.1) (i) and (6.3) (i).

We define subgroups $U_{i}$ of $S$ as follows:

$$
U_{i}=\left\{\left(a_{j k}\right) \in S \text { such that } a_{i+2} i+1=0\right\} .
$$


By definition $U_{0}=V, U_{n+2}=U$ and all these groups are $K_{0}$-invariant subgroups of $S$ which contain the commutator subgroup $S^{\prime}$ of $S$.

(6.4) (i) $K$ normalizes all $U_{i}(i=0,1, \cdots, n-2)$.

(ii) For $1 \leqq i \leqq n-3, N_{G}\left(U_{i}\right) \subseteq N_{G}(Z)$.

Proof. Let $V_{i}=\bigcap_{j \neq i} U_{j}$. Then each $V_{i} / S^{\prime}$ is an irreducible $K_{0^{-}}$ module by (2.7). It is easily seen from (2.7) that these modules $V_{i} / S^{\prime}$ are mutually nonisomorphic if $q>2$. Hence they are $K$-invariant. Since each group $U_{i}$ is a union of subgroups $V_{j}, U_{i}$ is $K$-invariant. This proves (i). If $1 \leqq i \leqq n-3, Z\left(U_{i}\right)=Z$ so that (ii) follows.

We are interested in the construction of a $(B N)$-pair in $G$. Define

$$
B=N_{G}(S)=S K \text {. }
$$

We will construct a subgroup $N$ to complete a $(B N)$-pair of $G$. We use terminology related to groups with a $(B N)$-pair. Thus, a subgroup which contains $B$ is called a parabolic subgroup. The group $N / K$ is to be the Weyl group. By assumption the group $H / O$ is a factor group of $\mathrm{GL}(n-2, q)$ by a subgroup in the center. So $H$ has a $(B N)$-pair. The idea is to extend this to a $(B N)$-pair of $G$. The linear groups involved in our discussion are groups of type $A_{l}$. Hence the Weyl groups are the symmetric groups. Let $W$ be the symmetric group of degree $l+1$. An ordered set of involutions $\left\{w_{1}, \cdots, w_{l}\right\}$ of $W$ is called a distinguished set of generators, if these elements generate $W$ and satisfy the relations

$$
\left(w_{i} w_{i+1}\right)^{3}=1 \text { and }\left(w_{i} w_{j}\right)^{2}=1 \quad \text { if }|i-j|>1 .
$$

(6.5) Assume that $q>2$. The $N_{G}(K)$ contains a set of involutions $\left\{t_{0}, t_{1}, \cdots, t_{n-2}\right\}$ such that

(a) $t_{i} \in N_{G}\left(U_{i}\right)$ for $i=0,1,2, \cdots, n-2$;

(b) the group $\left\langle K, t_{i}, i=0,1, \cdots, n-2\right\rangle / K$ is the symmetric group of degree $n$, and the ordered set $\left\{K t_{0}, K t_{1}, \cdots, K t_{n-2}\right\}$ is a distinguished set of generators; and

(c) $P=(P \cap V) \times Z^{x}$ and $R=(R \cap U) \times Z^{y}$, where $x=t_{n-2} t_{n-1} \cdots t_{1}$ and $y=t_{0} t_{1} \cdots t_{n-3}$.

Proof. Set $N_{0}=N_{G}(K) \cap N_{G}(Z), \quad N_{1}=N_{G}(K) \cap N_{G}(P)$ and $N_{2}$ $=N_{G}(K) \cap N_{G}(R)$. By assumption the pair $\left(B, N_{0}\right)$ is a $(B N)$-pair for $N_{G}(Z)$. It follows from (6.2) (iii) that $\left(B, N_{1}\right)$ is a $(B N)$-pair of $N_{G}(P)$. Similarly $\left(B, N_{2}\right)$ is a $(B N)$-pair of $N_{G}(R)$. For each $i, N_{G}\left(U_{i}\right)$ is a minimal parabolic subgroup of $G$. If $1 \leqq i \leqq n-3$, we have $N_{G}\left(U_{i}\right)$ $\subseteq N_{G}(Z)$ by (6.4) (ii). Since $q>2, N_{G}\left(U_{0}\right) \subseteq N_{G}(R)$ by (5.9). Similarly $N_{G}(U)$ is a subgroup of $N_{G}(P)$. 
By a theorem of Tits [40], $N_{G}\left(U_{i}\right) \cap N_{j}$ contains $K$ as a normal subgroup of index 2 , where $j=1$ or 2 according as $i \neq 0$ or $i \neq n-2$. Since $H$ has a $(B N)$-pair, $N_{H}\left(K_{0}\right) / K_{0}$ is isomorphic to the Weyl group. Hence we have $N_{0} / K \cong N_{H}\left(K_{0}\right) / K_{0}$. This implies that $N_{0}$ $=K N_{H}\left(K_{0}\right)$ and in particular $N_{H}\left(K_{0}\right)$ is contained in $N_{0}$. The structure of $H$ yields that $H$ contains an element $t_{i}(i=1,2, \cdots, n-3)$ which corresponds to the matrix

$$
\left(\begin{array}{lll}
I_{i} & & \\
& J & \\
& & I
\end{array}\right), \text { where } J=\left(\begin{array}{ll} 
& 1 \\
1 &
\end{array}\right) .
$$

It follows easily that $t_{i} \in N_{H}\left(K_{0}\right) \cap N_{H}\left(U_{i}\right)$. Since $N_{H}\left(K_{0}\right) \subseteq N_{0}$, we have

$$
N_{G}\left(U_{i}\right) \cap N_{j}=\left\langle K, t_{i}\right\rangle,
$$

where $i=1,2, \cdots, n-3$ and $j=0,1$ or 2 . Theorem of Tits asserts that the cosets $\left\{K t_{i}\right\}$ in the natural order form a distinguished set of generators for $N_{0} / K$. The group $N_{G}\left(U_{0}\right) \cap N_{2}$ contains a 2-element $t_{0}$. Either the ordered set $\left\{K t_{0}, \cdots, K t_{n-3}\right\}$ or the ordered set $\left\{K t_{1}, \cdots, K t_{n-3}, K t_{0}\right\}$ is a distinguished set of generators for $N_{2} / K$. $K$ leaves exactly two Sylow 2-groups of $N_{G}(V)$ by (6.3) (ii). Hence $Z_{1}=t_{0}^{-1} Z t_{0} \neq Z$ and $Z_{2}=t_{1}^{-1} Z_{1} t_{1} \neq Z_{1}$. If $\left(t_{0} t_{1}\right)^{2} \in K$, then

$$
Z_{2}=\left(t_{0} t_{1}\right)^{-1} Z t_{0} t_{1}=t_{0} Z t_{0}^{-1}=Z_{1} \text {, }
$$

a contradiction. Hence the ordered set $\left\{K t_{0}, K t_{1}, \cdots, K t_{n-3}\right\}$ is a distinguished set of generators for $N_{2} / K$. Similarly $N_{G}(U) \cap N_{1}$ contains a 2-element $t_{n-2}$ such that $\left\{K t_{1}, \cdots, K t_{n-2}\right\}$ is a distinguished set of generators for $N_{1} / K$. We will show that $\left(t_{0} t_{n-2}\right)^{2} \in K$. Set $t=t_{n-2}$.

Since $N_{G}(V) \subseteq N_{G}(T)$ by (3.2) (ii), we have $t_{0} \in N_{G}(T)$. Similarly $t \in N_{G}(T)$. By (6.3) (iv), $N_{G}(T)$ contains a normal subgroup $L$ of odd index such that $L / T$ is a direct product of two copies of $L_{2}(q)$. Let $L_{1}$ and $L_{2}$ be normal subgroups of $L$ such that $L / T=\left(L_{1} / T\right)$ $\times\left(L_{2} / T\right)$. We may label them suitably so that $L_{1} \cap S=U$. Since $t_{0}$ and $t$ are 2-elements, they are contained in $L$. As $t \in N_{G}(U)$, we have $t \in L_{2} U$. Write $t=l u$ with $l \in L_{2}$ and $u \in U$. Let $x$ be an element of $L_{1} \cap K$. Since $t$ normalizes $L_{1} \cap K$, we have

$$
[t, x] \in L_{1} \cap K \text {. }
$$

Since $l \in L_{2},[l, x]$ is an element of $T$. This implies that

$$
[u, x]=[l, x]^{-u}[t, x] \in T\left(L_{1} \cap K\right) \text {. }
$$


$L_{1} \cap K$ normalizes $U$. Hence $[u, x] \in U$. We conclude that

$$
[u, x] \in U \cap T\left(L_{1} \cap K\right)=T .
$$

Since $L_{1} / T \cong L_{2}(q)$ and $q>2$, this happens only when $u \in T$. Thus, $t \in L_{2}$. Similarly we have $t_{0} \in L_{1}$. Hence the commutator $\left[t_{0}, t\right]$ lies in $T$. By definition $\left[t_{0}, t\right] \in N_{G}(K)$. We have

$$
\left[T \cap N_{G}(K), K\right] \subseteq T \cap K=1 .
$$

By (5.1) we conclude that $T \cap N_{G}(K)=1$. Thus, $\left[t_{0}, t\right]=1$. Hence $\left(t_{0} t\right)^{2}=t_{0}^{2} t^{2} \in K$. Since $|K|$ is odd, we can choose involutions $t_{0}$ and $t_{n-2}$. If we set $y=t_{0} t_{1} \cdots t_{n-8}$, then by definition of $t_{i}$ we have $Z^{y} \subseteq R$ but $Z$ is not contained in $U$. Hence

$$
R=(R \cap U) \times Z^{y} .
$$

Similarly $P=(P \cap V) \times Z^{x}$ for $x=t_{n-2} \cdots t_{1}$. This proves (6.5).

Let $\left\{t_{i}, i=0,1,2, \cdots, n-2\right\}$ be a set of involutions of $N_{G}(K)$ which satisfies the conclusions of (6.5). Set

$$
N=\left\langle K, t_{i}, \quad i=0,1, \cdots, n-2\right\rangle .
$$

(6.6). Set $G_{0}=B N B$. Then $G_{0}$ is a subgroup of $G$ which has a $(B N)$ pair $(B, N)$ such that the Weyl group is the symmetric group of degree $n$.

Proof. We will prove that $G_{0}$ is a subgroup by showing $w B w w^{\prime} \subseteq G_{0}$ for any $w$ and $w^{\prime}$ of $N$. Use induction on the "length" of the expression of $w$ in terms of the distinguished generators $t_{0}, \cdots, t_{n-2}$. We may suppose that $w=r$ is one of the distinguished generators. We assume that $r \in N_{G}(P)$; the other case being similar. If $w^{\prime} \in N_{G}(P)$, then by a property of a $(B N)$-pair of $N_{G}(P)$ we have

$$
r B w w^{\prime} \subseteq B w^{\prime} B \cup B r w w^{\prime} B .
$$

It remains to prove that

$$
w S w^{\prime} \subseteq P w w^{\prime} S
$$

for any $w \in N_{1}=N_{G}(P) \cap N$ and any coset representative $w^{\prime}$ of cosets of $N_{1}$. By (6.5) (b), $N / K$ is the symmetric group of degree $n$. Hence 1 and $t_{0} t_{1} t_{2} \cdots t_{k}(k=0,1, \cdots, n-2)$ form a complete system of representatives. Since $N_{G}(P)$ has a $(B N)$-pair, we have a decomposition $S=P_{i} U_{i}(i=0,1, \cdots, n-3)$ such that $P_{0} \subseteq P, P_{0}$ is $K$-invariant and

$$
t_{i}^{-1} P_{i-1} t_{i}=t_{i-1}^{-1} P_{i} t_{i-1} \quad \text { for } 1 \leqq i \leqq n-3 .
$$


We have

$$
w S t_{0}=w P U_{0} t_{0}=P w t_{0} U_{0} .
$$

Suppose $k<n-2$. Use induction on $k$.

$$
\begin{aligned}
w S t_{0} \cdots t_{k} & \subseteq P w t_{0} \cdots t_{k-1} S t_{k} \\
& =P w t_{0} \cdots t_{k-1} P_{k} U_{k} t_{k} \\
& =P w t_{0} \cdots t_{k-1} P_{k} t_{k} U_{k} \\
& =P w P_{k}^{x} t_{0} \cdots t_{k} U_{k},
\end{aligned}
$$

where $x=t_{k-1} t_{k-2} \cdots t_{0}$. By $(6.7)$

$$
P_{k}^{x}=P_{k-1}^{y}, \quad \text { where } y=t_{k} t_{k-2} \cdots t_{0} .
$$

By (6.5) (b) we have

$$
y=u t_{k-2} \cdots t_{0} t_{k} \quad \text { where } u \in K .
$$

Since $P_{0}$ is $K$-invariant, every $P_{i}$ is also $K$-invariant. Hence

$$
P_{k-1}^{y}=P_{k-2}^{z}, \quad \text { where } \quad z=t_{k-1} t_{k-3} \cdots t_{0} t_{k} \text {. }
$$

We have $z=v t_{k-3} \cdots t_{0} t_{k-1} t_{k}$ with some $v \in K$. Finally we obtain

$$
P_{k}^{x}=P_{0}^{a}, \quad \text { where } s=t_{1} t_{2} \cdots t_{k} \text {. }
$$

This implies that $P_{k}^{x} \subseteq P$. Hence

$$
w S t_{0} s \subseteq P w P_{0}^{\circ} t_{0} s U_{k} \subseteq P w t_{0} s S .
$$

Set $t=t_{0} t_{1} \cdots t_{n-8}$ and consider $w S t t_{n-2}$. We have shown that $w S t \subseteq P w t S$. Since $S=Z^{t} U$ by (6.5) (c), we have

$$
\begin{aligned}
w S t t_{n-2} & \subseteq P w t S t_{n-2} \\
& =P w t Z^{t} U t_{n-2} \\
& =P w Z t t_{n-2} U \\
& =P w t t_{n-2} U .
\end{aligned}
$$

This completes the proof that the subset $G_{0}$ is a subgroup. We have in fact shown that $(B, N)$ is a $(B N)$-pair for $G_{0}$.

(6.8). Let $G_{0}=B N B$ be the subgroup defined in (6.6). Then $G_{0} \cong L_{n}(q)$.

Proof. This follows easily from either the theorem of Ito [21] or Tits [41]. Let $G_{1}$ be a minimal normal subgroup $\neq 1$ of $G_{0}$. If $x$ is any involution of $P, N_{G}(P)$ contains $C_{G}(x)$ by (5.8) and the assumption (6.1) (iii). Hence we conclude that $G_{1} \cap N_{G}(P) \neq 1$. By (5.8), $P$ is a minimal normal subgroup of $N_{G}(P)$. It is the unique one because $P$ 
is self-centralizing. Hence $P \subseteq G_{1} \cap N_{G}(P)$. It follows from the structure of $N_{G}(R),(5.10)$, that the normal subgroup of $N_{G}(R)$ generated by the conjugates of $P$ contains $S$. This implies that $S \subseteq G_{1}$. Hence we have $G_{0}=G_{1} B$. By a theorem of Tits [41], $G_{0}$ involves $L_{n}(q)$. Since $G_{0} / G_{1}$ is solvable, $G_{1}$ is isomorphic to $L_{n}(q)$. But $G_{0}$ and $L_{n}(q)$ have the same normalizer of $S$. We have $G_{0}=G_{1}$.

(6.9). Assume that $q=2$. There is a set of involutions $\left\{t_{i}, i=0,1\right.$, $2, \cdots, n-2\}$ such that the conditions (a), (b) and (c) of (6.5) (with $K=1$ ) are satisfied.

Proof. Since $q=2$, we have $n \geqq 6$ by the assumption (6.1) (iii). The following proof works in the case $n=5$, if we assume that the structure of $N_{G}(P)$ and $N_{G}(R)$ are as stated in (5.4) and (5.10). $N_{G}(P)$ is an extension of $P$ by $\operatorname{PGL}(n-1,2)$. Since a Sylow 2 -group $S$ splits over $P$, the extension of $N_{G}(P)$ over $P$ splits by a theorem of Gaschütz [12]. Since

$$
\operatorname{PGL}(n-1,2)=\mathrm{GL}(n-1,2)
$$

is the full group of automorphisms of $P, N_{G}(P)$ has a unique structure. We can identify $N_{G}(P)$ as the totality of matrices

$$
\left(\begin{array}{ll}
1 & \\
A & X
\end{array}\right)
$$

where $X$ range over $(n-1) \times(n-1)$ nonsingular matrices and $A$ range over all column vectors of size $n-1$. For each pair of integers $(i, j)$, let $e_{i j}$ be the $n \times n$ matrix whose $(i, j)$ entry is 1 and whose other entires are all zero. Set

$$
x_{i j}=I_{n}+e_{i j} \quad(i>j) .
$$

Then $x_{i j}$ is an element of $S$. Define

$$
t_{i}=\left(\begin{array}{ccc}
I_{i} & & \\
& J & \\
& & I
\end{array}\right), \quad J=\left(\begin{array}{ll} 
& 1 \\
1 &
\end{array}\right)
$$

for $i=1,2, \cdots, n-2$. Set $t=t_{n-2}$.

By (6.3) (iv), $N_{G}(T) / T$ is a direct product of two copies of $L_{2}(2)$. One factor contains $U / T$ and the other $V / T$. The element $T t$ belongs to the factor containing $V / T$ because $t$ is conjugate to $x_{n n-1}$. We will find an element $s=t_{0}$ in the normal subgroup of $N_{G}(T)$ generated by $U$. It is obvious that there is an element $s$ of $N_{G}(V)$ such that 
(1) $x_{n 1}^{s}=x_{n 2}$, and

(2) $s$ is conjugate to $x_{21}$ in $N_{G}(V)$.

Since $s$ is contained in the normal subgroup generated by $U$, we have

(3) the commutator $[s, t]$ lies in $T$.

We need some properties of the group $Q$ consisting of all matrices of the form

$$
\left(\begin{array}{ll}
I & \\
X & I
\end{array}\right)
$$

where $X$ range over all matrices of size $(n-2,2) . Q$ is an elementary abelian group, which admits a group of automorphisms which consists of matrices

$$
\left(\begin{array}{ll}
I_{2} & \\
& Y
\end{array}\right)
$$

We summarize properties of $Q$ which will be needed.

(6.10) (i) $Q$ is a normal subgroup of $N_{G}(V)$.

(ii) $Q$ contains precisely four conjugate classes of involutions in $N_{G}(P)$, which are represented by $x_{n 1}, x_{n 2}, x_{n 1} x_{n 2}$ and $x_{n-1} x_{n 2}$.

(iii) $Q$ contains three subgroups $Q_{1}, Q_{2}$, and $Q_{3}$ of order $2^{n-2}$ such that involutions of $Q_{i}(i=1,2,3)$ are central and any central involution of $Q$ is contained in one of $Q_{i}$.

Proof. Let $T_{0}$ be the subgroup of $T$ consisting of matrices $\left(a_{i j}\right)$ with $a_{21}=a_{31}=a_{32}=a_{n-1 n-2}=a_{n n-2}=a_{n n-1}=0$. Then the method used in the proof of (2.12) yields the following result. Any abelian normal subgroup of $T$ which is not contained in $T_{0}$ has order at most $q^{2(n-2)}$, and there are precisely two elementary abelian normal subgroups of order $q^{2(n-2)}$ which are not contained in $T_{0}$. This implies in particular $T_{0}$ is a characteristic subgroup of $T$. Since $S$ normalizes $Q$ and $N_{G}(V) \subseteq N_{G}(T)$ by (3.2), the assertion (i) follows easily.

Set $Q_{1}=Q \cap P, \quad Q_{2}=\left\langle x_{i 2} \quad i=3, \cdots, n\right\rangle$ and $Q_{3}=\left\langle x_{i 1} \quad x_{i 2} \quad i=3\right.$, $4, \cdots, n\rangle$. Then $N_{G}(Q) \cap N_{G}(P)$ normalizes all $Q_{i}$, and acts transitively on $Q_{i}-1$. Furthermore the stabilizer of $x_{n 2}$ is transitive on $Q_{1}-Z$. This proves (ii). Since $x_{n 1}^{s}=x_{n 2}, x_{21} x_{n 2} x_{21}=x_{n 1} x_{n 2}$, elements of $Q_{i}-1$ are central and $Q$ contains no other central involution. This establishes (6.10).

By (6.10) (i), $s$ normalizes $Q$. By (2), $s$ is conjugate to $x_{21}$ in $N_{G}(V)$. Hence $C_{Q}(s)$ is conjugate to $C_{Q}\left(x_{21}\right)$. Thus involutions of $C_{Q}(s)$ are central. By (6.10) (iii), we have $Q_{1}^{s}=Q_{2}$ in the notation used in the 
proof of (6.10). For each $i=3,4, \cdots, n, x_{i 1}^{s} \in Q_{2}$ and $x_{i 1} x_{i 1}^{s} \in C_{Q}(s)$. Hence we have

(4) $x_{i 1}^{s}=x_{i 2}$ for $i=3,4, \cdots, n$.

We prove that there is an element $s$ of $N_{G}(V)$ which satisfies the conditions (1) through (4) and, in addition,

(5) $x_{n j}^{s}=x_{n j}$ if $j \geqq 3$.

Since $N_{G}(V) \subseteq N_{G}(R), s$ normalizes $R$. By (2), $s$ acts in $R$ as a transvection. Hence for each $j=3,4, \cdots, n-1$, we have

$$
x_{n j}^{*}=x_{n j} \text { or } x_{n j} x_{0}
$$

where $x_{0}=x_{n 1} x_{n g}$. Choose a minimal integer $k$ such that $\left[x_{n k}, s\right]=x_{0}$. Replace $s$ by $s^{\prime}=x_{k 1}^{-1} s x_{k 1}$. The element $s^{\prime}$ satisfies (1)-(4). In addition we have $\left[x_{n j}, s^{\prime}\right]=1$ if $3 \leqq j \leqq k$. This process may be repeated. Therefore there is an element $s$ satisfying all conditions (1)-(5).

We prove next that the conditions (1)-(5) imply

(6) $\left[x_{i j}, s\right]=1$ if $3 \leqq j<i$.

Set $x=x_{i j}(3 \leqq j<i)$ and $y=x_{n k}(k \geqq 3)$. By the commutator identity we have

$$
[x, y, s]^{\nu}[y, s, x]^{\bullet}[s, x, y]^{x}=1 .
$$

Here we omit the exponent -1 on the middle term of commutators since every element is an involution. Since $j, k \geqq 3$, the first two commutators vanish by (5). Hence we have

$$
[s, x, y]=1 \text {. }
$$

This yields that $[s, x] \in R$. Take $u=x_{\imath \mathfrak{l}}$. Then the commutator identity implies

$$
[s, x, u]=1 \text {. }
$$

Hence we have $[s, x] \in Z(V)$. If $z=[s, x] \neq 1, x$ is conjugate to $z x_{n-1} 3$ in $N_{G}(P)$. This is a contradiction because $z x_{n-1}$ is conjugate to $x_{n-11} x_{n 2}$ which is not central by (3.4).

There is an element $s$ which satisfies

(7) $[s, t]=1$ in addition to the conditions (1)-(6).

Let $s$ be an element which satisfies (1)-(6). Set $u=[s, t]$. We claim that $u \in Z(T)$. By (3), $u$ belongs to $T$. If $x=x_{i 1}(i \leqq n-2)$, we have

$$
x^{u}=x^{s t s t}=y^{t s t}=y^{s t}=x
$$

where $y=x_{i 2}$ by (4). Similarly $y^{u}=y$. Hence $u \in C_{G}(Q)=Q$. If $j \geqq 3$,

$$
x_{n j}^{u}=x_{n j}^{t s t}=x_{n-1 j}^{s t}=x_{n j}
$$


by (5) and (6). This proves that $u \in Z(T)$. By definition $u=[s, t]$ $=(s t)^{2}$ and $u$ commutes with both $s$ and $t$. Hence either $u=1$ or $u=x_{n 1} x_{n-1} x_{n 2} x_{n-1}$. In the latter case replace $s$ by $s^{\prime}=x_{n 1}^{-1} s x_{n 1}$. This element $s^{\prime}$ satisfies (1)-(6), and

$$
\begin{aligned}
{\left[s^{\prime}, t\right] } & =\left(s^{\prime} t\right)^{2}=x_{n 1} x_{n 2}(s t)^{2} x_{n-1} x_{n-12} \\
& =1 .
\end{aligned}
$$

Set $t_{0}=s$ for an element $s$ which satisfies (1)-(7). Conditions (a) and (c) are obviously met. Considering the actions on $R$, we see that $\left(s t_{1}\right)^{3}$ and $\left(s t_{i}\right)^{2}(i=2, \cdots, n-3)$ centralize $R$. Since $R$ is self-centralizing, they are contained in $R$. If $i \neq n-3, t$ commutes with $t_{i}$. Hence by (7), $t$ commutes with $s t_{i}$. Since $R \cap R^{t}=1$, we have

$$
\left(s t_{1}\right)^{3}=\left(s t_{i}\right)^{2}=1
$$

except possibly $i=n-3$. Set $\left(s t_{n-3}\right)^{2}=r$ and prove that $r=1$. We have $x^{r}=x$, where $x=x_{n-11}$. This implies that $r$ is an element of $T$. Hence $r^{t} \in T$. We have

$$
r^{t}=\left(t s t_{n-3} t\right)^{2}=\left(s t_{n-3} t t_{n-3}\right)^{2},
$$

because $\left(t t_{n-3}\right)^{3}=1$. Hence

$$
\begin{aligned}
r^{t} & =\left(t_{n-3} r s t t_{n-3}\right)^{2} \\
& =t_{n-3}(r s t)^{2} t_{n-3} \\
& =r v
\end{aligned}
$$

where $v=t_{n-8} r^{t s} t_{n-3}$. We have shown that $r$ is an element of $R$. Hence if $r=\left(a_{i j}\right)$, we have $a_{i j}=0$ if $i \neq j$ and $i \neq n$. Hence only nonzero entries of $r^{t}$, off diagonal, are in the second row from the bottom. By (4) and (6) the same is true for $r^{t s}$. Hence the last two rows of $v$, except on the main diagonal, contain zero only. Since $v=r r^{t}$, we have $r=1$. This proves (6.9).

Propositions (6.6) and (6.8) yield that $G$ contains a subgroup $G_{0}$ which is isomorphic to $L_{n}(2)$. The subgroup $G_{0}$ is the one generated by $S$ and $t_{i}(i=0,1, \cdots, n-2)$.

7. Proof of the equality $G=G_{0}$. In the preceding section we constructed a subgroup $G_{0}$ which is isomorphic to $L_{n}(q)$. In this section we will show that $G=G_{0}$.

We may consider $G_{0}$ as the factor group of $\operatorname{SL}(n, q)$ by the center. In this way each element of $G_{0}$ is represented by an $n \times n$ matrix over $F$. We use the notation of (2.6), (2.8) and (2.9). Thus, $S$ is a Sylow 2 -group of $G_{0}$ which is represented by the elements (2.6). We may 
assume that $G_{0}$ was constructed from $N_{G}(S)$. By construction $G_{0}$ contains several local subgroups of $G$.

(7.1) $G_{0}$ contains $N_{G}(Z)$. Furthermore if $X$ is a subgroup of $S$ such that $T \subseteq X \subseteq S$, then $N_{G}(X) \subseteq G_{0}$.

PROOF. The constructions (6.5) and (6.9) show that $N_{G}(Z) \subseteq G_{0}$. The involution $t_{0}$ is an element of $N_{G}(V)$ which does not normalize $S$. By (6.3) (ii) we have

$$
N_{G}(V)=\left\langle S, K, t_{0}\right\rangle .
$$

Similarly $N_{G}(U)=\left\langle S, K, t_{n-2}\right\rangle$. Hence $G_{0}$ contains $N_{G}(U)$ and $N_{G}(V)$. This implies that $G_{0}$ contains $N_{G}(T)$, because $N_{G}(T)$ is generated by $N_{G}(U)$ and $N_{G}(V)$. The rest of the assertion follows from (3.2) (ii).

Any conjugate class of involutions of $G_{0}$ contains an element which is represented by the element $j_{l}$ of $(2.2)$. Let $C_{l}$ denote the conjugate class of involutions of $G_{0}$ which is represented by $j_{l}$. If $z$ is an involution of $Z$, then $z \in C_{1}$.

(7.2) Let $j$ be the involution of $S$ which is represented by $j_{l}$ of (2.2). Set $W=C_{s}(j)$. Then we have $N_{G}(W) \subseteq N_{G}(Z)$. by

Proof. Let $Y$ be the totality of elements of $S$ which are represented

$$
\left(\begin{array}{lll}
I & & \\
& I & \\
\lambda I_{l} & & I
\end{array}\right) \quad(\lambda \in F) .
$$

Then the center of $W$ is the direct product $Y \times Z$. Let $Z_{2}(W)$ denote the second center of $W$. A simple computation shows that

$$
\left[W, Z_{2}(W)\right]=Z, \quad \text { if } l \geqq 3 .
$$

Hence $Z$ is a characteristic subgroup of $W$ if $l \geqq 3$. This proves (7.2) for $l \geqq 3$. The same containment holds in the case $l=2$ because $Z$ does not fuse in $Z(W)-Z$ by (3.4).

(7.3) Let $z$ be a central involution which is contained in $G_{0}$. The following hold:

(i) $z \in C_{1}$;

(ii) If $z^{x} \in G_{0}$ for some $x$ of $G$, then $x \in G_{0}$, in particular $C_{G}(z) \subseteq G_{0}$.

Proof. By assumption $z$ is conjugate to $j_{1}$ in $G$. Suppose that $z \in C_{i}$ for some $i \geqq 2$. Then $j_{1}$ is conjugate to $j_{i}$ in $G$. By (2.17) (i), $W=C_{S}\left(j_{i}\right)$ is a Sylow 2-group of $C_{G}\left(j_{i}\right) \cap G_{0}$. Let $S^{\prime}$ be a Sylow 2-group of $C_{G}\left(j_{i}\right)$ which contains $W$. Then we have $G_{0} \cap S^{\prime}=W$. Since $S^{\prime}$ contains $W$ 
as a proper subgroup, $N_{G}(W)$ is not contained in $G_{0}$. This contradicts (7.2). Thus $z \in C_{1}$. We may assume that $z=j_{1}^{w}$ for some $w \in G_{0}$.

Suppose that

$$
z^{x}=j_{1}^{w x} \in G_{0}
$$

By (i), $z^{x} \in C_{1}$ so $z^{x}=j_{1}^{\nu}$ for some $y \in G_{0}$. This implies that $w x y^{-1}$ $\in C_{G}\left(j_{1}\right)$. By (7.1), $C_{G}\left(j_{1}\right)$ is contained in $G_{0}$. Since both $w$ and $y$ are elements of $G_{0}$, we conclude that $x \in G_{0}$.

(7.4) Let $Q$ be a 2-subgroup of $G$. If $Q \cap G_{0}$ contains a central involution, then $Q$ is contained in $G_{0}$.

Proof. Let $X$ be a Sylow 2-group of $G$ which contains $Q$. By assumption $Q \cap G_{0}$ contains a central involution $z$. Since $Q \subseteq X, z$ centralizes $Z(X)$. By (7.3) (ii), $Z(X) \subseteq G_{0}$. Since $Z(X)$ contains a central involution, the second application of (7.3) (ii) yields that $X \subseteq G_{0}$.

(7.5) Let $j$ be an involution of $G_{0}$ which belongs to the class $C_{2}$. Then any 2-element of $C_{G}(j)$ lies in $G_{0}$.

This is the key result. The proof differs according as $q=2$ or not. In (7.5) we assume that $q>2$. Suppose (7.5) is false, and prove a series of lemmas.

(a) Let $W^{\prime}$ be a Sylow 2-group of $C_{G}(j)$ such that $W^{\prime}$ is not contained in $G_{0}$ and with this restriction the order of $W^{\prime} \cap G_{0}$ is maximal. Set $I=W^{\prime} \cap G_{0}$. Then $j \in I$ and $I \cap C_{1}$ is empty.

Proof. Clearly $j \in I$. Since $W^{\prime}$ is not contained in $G_{0}, I \cap C_{1}$ is empty by (7.4).

(b) Set $J=I C_{G}(I)$ where $I$ is the subgroup defined in (a). There exists a Sylow 2-group $W_{1}$ of $C_{G}(j) \cap G_{0}$ such that $J_{1}=J \cap W_{1}$ is a Sylow 2-group of $J$.

Proof. Let $S^{\prime}$ be a Sylow 2-group of $G_{0}$ which contains $I$. Then $Z\left(S^{\prime}\right)$ centralizes $I$ but is not contained in $I$ by (a). Let $J_{1}$ be a Sylow 2-group of $J$ which contains $I Z\left(S^{\prime}\right)$, and let $W_{1}$ be a Sylow 2-group of $C_{G}(j)$ which contains $J_{1}$. Then $G_{0} \cap W_{1}$ contains $I Z\left(S^{\prime}\right)$ and (7.4) yields that $W_{1} \subseteq G_{0}$.

At this stage we may assume that

$$
j=j_{2} \text { and } W_{1}=C_{S}\left(j_{2}\right)
$$

by choosing suitable conjugates, if necessary. Set $W=C_{S}\left(j_{2}\right)$.

(c) $J / I$ is a (TI)-group. 
Proof. Set $J_{1}=J \cap W$. Then $J_{1}$ is a Sylow 2-group of $J$. Choose a Sylow 2-group $J_{2}$ of $J$ such that $J_{1} \cap J_{2} \neq I$. If $W_{2}$ is a Sylow 2-group of $C_{G}(j)$ containing $J_{2}, G_{0} \cap W_{2}$ has larger order than $I$. This implies that $W_{2} \subseteq G_{0}$. Hence $W_{2}$ contains the maximal normal 2-subgroup of $C_{G}(j) \cap G_{0}$. It follows from the structure of $G_{0}$ that $Z(S) \subseteq W_{2}$. Thus $Z \subseteq W_{2} \cap J=J_{2}$.

Let $J_{0}$ be a Sylow 2-group of $J$ which contains $I Z\left(S^{\prime}\right)$. Here $S^{\prime}$ is a Sylow 2-group of $G$ which contains $W^{\prime}$. If $J_{1} \cap J_{0} \neq I$, then $J_{0}$ contains $Z I$. Hence $J_{0}$ would be a subgroup of $G_{0}$. This contradicts the definition of $S^{\prime}$. Hence $J_{1} \cap J_{0}=I$. Since $q>2,(2.20)$ is applicable and yields that $J / I$ is a $(T I)$-group.

The structure theorems of (TI)-groups [34] yields the existence of a cyclic group $C$ of odd order of $J$ which normalizes the Sylow 2-group $J_{1}$ of $J$ and acts transitively on the set of involutions of $J_{1} / I$.

(d) $C$ is a subgroup of $C_{G}(I) \cap G_{0}$.

Proof. Since $C$ is odd, we have $C \subseteq C_{G}(I)$. Let $x$ be a generator of $C$. Then $W^{x}$ contains $J_{1}$. Hence $W^{x} \subseteq G_{0}$. There is an element $y$ of $C_{G}(j) \cap G_{0}$ such that $W^{x}=W^{y}$. Then $x y^{-1}$ is an element of $N_{G}(W)$. By (7.2) we have $x y^{-1} \in G_{0}$. This implies $x \in G_{0}$.

(e) $j$ is not contained in $C_{2}$.

Proof. By (d), $C$ is a subgroup of $C_{G}(j) \cap G_{0}$. This implies that $C$ normalizes $Z(T)$. This is so because $Z(T)$ is the center of the maximal 2-subgroup of $C_{G}(j) \cap G_{0}$. The group $C_{G}(j) \cap G_{0}$ induces in $Z(T)$ the group of automorphisms which is isomorphic to $L_{2}(q)$. Hence $C$ induces a cyclic group of automorphisms of order at most $q+1$. Thus the group $J_{1} / I$ contains at most $q+1$ involutions. Since $J_{1} / I$ contains $Z I / I$, we conclude that $Z I / I$ contains all the involutions of $J_{1} / I$. Take a generator $x$ of $C$. Suppose that $Z^{x} \neq Z$. Then $Z^{x} \subseteq Z I$ and $Z \cap Z^{x}=1$. Hence $\left|Z Z^{x} \cap I\right|=q$. Since $x$ centralizes $I$, we conclude that $x$ normalizes $Z Z^{x}$. Thus $Z Z^{x}$ is a subgroup of $Z(T)$ which contains at least $q-1$ conjugates of $Z$ and at least $q-1$ noncentral involutions. If $q>2, Z(T)$ contains no such subgroup. Hence $C$ normalizes $Z$. This implies that $C$ normalizes $Z(T) \cap P$ and $Z(T) \cap R$. Since $|C|$ is odd, there is a subgroup $X$ of $Z(T) \cap P$ such that $X$ is $C$-invariant and $Z(T) \cap P=X \times Z$. Similarly there is a $C$-invariant subgroup $Y$ such that

$$
Z(T) \cap R=Y \times Z .
$$

The group $\langle X, Y, Z\rangle$ contains $Z(W)$. Since $Z(W) \subseteq C_{W}(I), Z(W)$ is contained in $Z I$. Hence $|Z(W) \cap I|=q$. Since $C$ acts trivially on 
$Z(W) \cap I$, we conclude that $C$ acts trivially on either $X$ or $Y$, say $X$ (in fact trivial on both). By (d), $C$ centralizes $I$. Hence $C$ centralizes the commutator $[X, I]$. Since $I \subseteq S$, this commutator is contained in $Z$. On the other hand $C$ acts transitively on the set of involutions of $Z$. Hence we obtain $[X, I]=1$; in other words, $X \subseteq C_{W}(I)$. Since $Z I / I$ contains all the involutions of $J_{1} / I, Z I$ contains $X$. Hence $Z X \subseteq Z I$. This implies that $|Z X \cap I|=q$. By definition we have $Z X=Z(T) \cap P$. Therefore any involution of $Z X$ is central. Thus $I \cap Z X$ contains a central involution. This contradicts with (a).

(7.6) The proposition (7.5) holds even when $q=2$.

Proof. Again we suppose that (7.6) is false and prove a series of lemmas. Set $W=C_{s}\left(j_{2}\right)$ as before.

(a) There is a subgroup $T^{\prime}$ of $C_{G}(j)$ which is conjugate to $T$, but not contained in $G_{0}$.

Proof. By assumption there is a Sylow 2-group $W^{\prime}$ of $C_{G}(j)$ which is not contained in $G_{0}$. Then $W^{\prime}=W^{x}$ for some element $x$. If $T^{x} \subseteq G_{0}$, then $W^{x}$ would be contained in $G_{0}$ by (7.4).

(b) Let $T^{\prime}$ be a subgroup of $C_{G}(j)$ such that $T^{\prime}$ is conjugate to $T, T^{\prime}$ is not contained in $G_{0}$ and with these restrictions the order $T^{\prime} \cap G_{0}$ is maximal. There is a Sylow 2-group $W^{\prime}$ of $C_{G}(j)$ which contains $T^{\prime}$. If possible, let $T^{\prime}$ be chosen so that

$$
W^{\prime} \cap G_{0} \neq T^{\prime} \cap G_{0} .
$$

Set $I=T^{\prime} \cap G_{0}, \quad I_{0}=W^{\prime} \cap G_{0}, \quad J=I C_{G}(I)$ and $J_{0}=I_{0} C_{G}(I)$. Then $I_{0} \cap C_{1}$ is empty.

Proof. This is mostly a collection of definitions. The last assertion follows from (7.4).

(c) There is a Sylow 2-group $W_{1}$ of $C_{G}(j) \cap G_{0}$ such that $J_{1}=J \cap W_{1}$ is a Sylow 2-group of $J$.

Proof is the same as (7.5) (b).

(d) There is a conjugate subgroup of $T$ in $C_{G}(j) \cap G_{0}$ which contains $I$.

Proof. By (c), $G_{0} \cap C_{G}(j)$ contains a Sylow 2-group $W_{1}$ such that $J_{1}=J \cap W_{1}$ is a Sylow 2-group of $J$. By definition, $K=I Z\left(T^{\prime}\right)$ is a subgroup of $J$ with order larger than $|I|$. There is an element $x$ of $J$ such that $K^{x} \subseteq J_{1}$. Then $T^{\prime \prime}=x^{-1} T^{\prime} x$ satisfies

$$
K^{x} \subseteq J_{1} \cap T^{\prime \prime} \subseteq G_{0} \cap T^{\prime \prime} .
$$


Since $|I|<\left|K^{x}\right|$, we conclude that $T^{\prime \prime} \subseteq G_{0}$. Since $x \in J$, we have $I \subseteq T^{\prime \prime}$ and $T^{\prime \prime} \subseteq C_{G}(j) \cap G_{0}$.

We assume that $I \subseteq T \subseteq C_{G}(j) \cap G_{0}$. This is possible by choosing suitable conjugate elements.

(e) $C_{G}(j) \cap G_{0}$ normalizes $Z(T)$ and induces a group of automorphisms, which is isomorphic to $L_{2}(2)$.

Proof. By assumption $j$ is a noncentral involution of $Z(T)$. Hence $Z(T)$ is the center of the maximal normal 2-subgroup of $C_{G}(j) \cap G_{0}$. So $C_{G}(j) \cap G_{0}$ normalizes $Z(T)$.

The structure of $G_{0}$ yields that the group induced in $Z(T)$ is isomorphic to $L_{\mathbf{2}}(q)$.

(f) The group $J_{0} / I$ contains a normal subgroup of index one or two, which is a (TI)-group. In this (TI)-group, a Sylow 2-group contains more than one involution.

Proof. We assume that $I=I_{0}$ for all admissible conjugate subgroups of $T$. This assumption implies that, if $W^{\prime \prime}$ is a Sylow 2-group of $C_{G}(j)$, which is not contained in $G_{0}$, the order of $W^{\prime \prime} \cap G_{0}$ is at most $|I|$. Suppose that $J_{2}$ is a Sylow 2-group of $J$ with $J_{1} \cap J_{2} \neq I$. If $W_{2}$ is a Sylow 2-group of $C_{G}(j)$ containing $J_{2}$, we have

$$
I \subset J_{1} \cap J_{2} \subseteq G_{0} \cap W_{2} .
$$

Hence $W_{2}$ is a subgroup of $G_{0}$. This yields that $W_{2}$ contains $Z(T)$ by (e). Hence $Z(T) \subseteq J_{2}$. Since $I \subseteq T, Z(T)$ is contained in $J$. The group $I Z(T) / I$ has at least order 4 because $Z(T) \cap P \cap I=1$ by (b). Hence (2.20) is applicable and yields the result.

Suppose that $I \neq I_{0}$. Let $K$ be a Sylow 2-group of $J_{0} \cap G_{0}$ which contains $I_{0}$. Then $K \subseteq G_{0} \cap C_{G}(j)$. Thus $K$ normalizes $Z(T)$ by (e). Set $K_{1}=C_{K}(Z(T))$. By (e), we have $\left|K: K_{1}\right| \leqq 2$. The group $T$ is a Sylow 2-group of $C_{G}(Z(T))$. Hence $K_{1}$ is contained in a conjugate subgroup $T_{1}$ of $T$. We have $T_{1} \subseteq G_{0} \cap C_{G}(j)$.

Suppose that $K=K_{1}$. Then $K \subseteq T_{1}$. Since $I_{0}=G_{0} \cap W^{\prime}$, there is an element $x$ of $W^{\prime}$ which normalizes $I_{0}$ but is not contained in $G_{0}$. There is a central involution $z$ in $K$. By (7.3) (ii), $z^{x}$ is not contained in $G_{0}$. Hence

$$
I_{0} \subseteq K^{x} \subseteq T_{1}^{x} \text { and } T_{1}^{x} \subseteq C_{G}(j),
$$

but $T_{1}^{x}$ is not contained in $G_{0}$ because $z^{x} \in T_{1}^{x}$ and $z^{x} \notin G_{0}$. This contradicts the definition of $I$. Hence $K \neq K_{1}$. In fact, the above argument proves that $K_{1}$ does not contain $I_{0}$. Similarly no conjugate subgroup of $I_{0}$ in $J_{0}$ is contained in $K_{1}$. By a lemma of Thompson [39, Lemma 
(5.38)], $J_{0}$ contains a normal subgroup $L$ of index 2 such that $K \cap L$ $=K_{1}$.

Let $K_{2}$ be a Sylow 2-group of $L$ such that $K_{1} \cap K_{2} \neq I$. Then $K_{2}$ is contained in a conjugate subgroup $T_{2}$ of $T$ which lies in $C_{G}(j)$. Since $G_{0} \cap T_{2}$ has a larger order than $I, T_{2}$ is contained in $G_{0}$. Thus $T_{2} \subseteq$ $G_{0} \cap C_{G}(j)$ and $T_{2}$ contains $Z(T)$. Proposition (2.20) yields that $L / I$ is a (TI)-group. Since $K$ contains $Z(T), K / I$ contains at least 3 involutions.

(g) $Z(T) \cap I$ is a group of order 4 .

Proof. By (f), $J_{0} / I$ contains a $(T I)$-group $L / I$. By (c), there is a Sylow 2-group $L_{2}$ of $L$ which is contained in $G_{0}$. The structure theorem of (TI)-groups [34] yields the existence of a cyclic group $C$ of odd order such that $C$ normalizes $L_{2}$ and acts regularly on the set of involutions of $L_{2} / I$. Let $W_{1}$ be a Sylow 2-group of $G_{0} \cap C_{G}(j)$ which contains $L_{2}$, and let $T_{1}$ be a conjugate subgroup of $T$ contained in $W_{1}$. Then $T_{1} \cap L_{2}$ has a larger order than $I$. If $x$ is a generator of $C, T_{1}^{x} \cap G_{0}$ has a larger order than $I$. Hence by definition of $I$, we conclude $T_{1}^{x} \subseteq G_{0}$ and $W_{1}^{x} \subseteq G_{0}$. As in (7.5) (d), we have $x \in G_{0}$. Hence $C \subseteq$ $G_{0} \cap C_{G}(j)$. By (e), $C$ normalizes $Z(T)$ and induces a group of automorphisms of $Z(T)$, a subgroup of $L_{2}(2)$. Since $C$ acts regularly on the set of involutions of $L_{2} / I$, the order of $C$ must be three and

$$
|Z(T): Z(T) \cap I|=4 .
$$

This proves the proposition $(\mathrm{g})$.

(h) Set $Z_{1}=Z(T) \cap I$.

(1) $T$ is a Sylow 2-group of $C_{G}\left(Z_{1}\right)$.

(2) If $x$ is an element of $C_{G}\left(Z_{1}\right)$, then

$$
Z(T)^{x} \cap G_{0} \subseteq Z(T) .
$$

Proof. Since $Z_{1}$ is a group of order 4 by $(\mathrm{g})$, the first assertion follows from the structure of $G_{0}$. Suppose that the element $j$ (which is in $\left.Z_{1}\right)$ is a product of two central involutions $u$ and $v$ of $C_{G}\left(Z_{1}\right) \cap G_{0}$. Then $u$ and $v$ are represented in $G_{0}$ by a pair of commuting transvections whose product represents $j$. There are exactly 3 such pairs. Hence both $u$ and $v$ are contained in $Z(T)$. If $x$ is an element of $C_{G}\left(Z_{1}\right)$ and if $Z(T)^{x} \cap G_{0}$ is not $Z_{1}$, then there is a pair of central involutions of $Z(T)^{x}$ whose product is $j$. This implies that $Z(T)^{x} \cap G_{0}$ $\subseteq Z(T)$.

(i) We have $T=Z(T) I$.

Proof. Let $z$ be a central involution which lies in $T-Z(T)$. By 
definition there is a Sylow 2-group $T_{1}$ of $C_{G}\left(Z_{1}\right)$ such that $I=G_{0} \cap T_{1}$. Let $z_{1}$ be a central involution of $Z\left(T_{1}\right)$. Then $z_{1}$ is not contained in $G_{0}$ by (7.4). By (h), the coset $z Z_{1}$ is not conjugate to $z_{1} Z_{1}$ in $C_{G}\left(Z_{1}\right) / Z_{1}$. Hence there is an involution $w Z_{1}$ of $C_{G}\left(Z_{1}\right) / Z_{1}$ such that $w Z_{1}$ commutes with both $z Z_{1}$ and $z_{1} Z_{1}$, and $w z Z_{1}$ is conjugate to either $z Z_{1}$ or $z_{1} Z_{1}$. Suppose that $w z Z_{1}$ is conjugate to $z Z_{1}$. Then $w z_{1} Z_{1}$ is conjugate to $z_{1} Z_{1}$. By (h) applied to a conjugate subgroup of $G_{0}$ containing $C_{G}\left(z_{1}\right)$, we conclude that $w z_{1} \in Z\left(T_{1}\right)$. We remark that the coset $w z_{1} Z_{1}$ contains a unique noncentral involution of $G$ and hence $z_{1}$ centralizes every element of $w z_{1} Z_{1}$. Since $w z_{1} \in Z\left(T_{1}\right)$, we have $w \in Z\left(T_{1}\right)$. On the other hand, the coset $z Z_{1}$ contains a unique central involution. Hence $w$ centralizes $z$. This implies $w \in G_{0}$. So we have

$$
w \in G_{0} \cap Z\left(T_{1}\right)=Z_{1} .
$$

This is not the case. Hence the coset $w z Z_{1}$ is conjugate to $z_{1} Z_{1}$. Since $z$ centralizes $w z Z_{1}, w z Z_{1}$ is contained in $G_{0}$. By (h) we have $w z \in Z(T)$, which implies $w \in T$. Since $w$ centralizes $z_{1}$, we have $w \in C_{T}\left(z_{1}\right)$. By definition $z_{1}$ centralizes $I$. The maximality of $I$ forces

$$
I=G_{0} \cap C_{G}\left(Z_{1}\right) \cap C_{G}\left(z_{1}\right) .
$$

This implies that $C_{T}\left(z_{1}\right)=I$ and $w \in I$. Therefore

$$
z=w^{-1} w z \in I Z(T) .
$$

This is true for any central involution $z$ of $T-Z(T)$. Hence $T=I Z(T)$ because $T$ is generated by central involutions.

(j) $I \cap C_{1}$ is not empty.

Proof. Since $n \geqq 5, T$ is different from $Z(T)$. In fact, $T$ contains a central involution $z$ which is represented by the matrix

$$
E+e_{n-21}
$$

where $E$ is the identity matrix and $e_{n-21}$ is the matrix with 1 at the $(n-2,1)$ place and zero elsewhere. By $(\mathrm{i})$,

$$
\langle Z(T), z\rangle \cap I
$$

contains an element $w$ represented by a matrix $\left(a_{i j}\right)$ whose entries below the main diagonal are zero except possibly $a_{n-2}, a_{n-1}, a_{n-1}$, $a_{n 1}, a_{n 2}$ and $a_{n-21}=1$. Then for some element $u$ of $Z_{1}, u w$ is represented by a transvection and $u w \in C_{1} \cap I$.

This lemma contradicts with (b) and the proposition (7.6) is proved. 
(7.7) Elements of $C_{2}$ do not fuse in $C_{i}$ for $i \neq 2$.

This is proved in the same way as (7.3).

(7.8) Let $u$ be an involution of $G_{0}$ which belongs to the class $C_{3}$. Then any 2-element of $C_{G}(u)$ is contained in $G_{0}$.

Proof. We may assume that $u=j_{8}$ where $j_{3}$ is given by (2.2). Then there exists a central involution $z$ of $G_{0}$ such that $z u \in C_{2}$. We have $j_{1} u \in C_{3}$. Elements of $C_{3}$ do not fuse in $C_{2}$ by (7.7). Hence $z$ is not conjugate to $j_{1}$ in $C_{G}(u)$.

Suppose that (7.8) is false. Then there is a Sylow 2-group $W_{1}$ of $C_{G}(u)$ which is not contained in $G_{0}$. Let $S_{1}$ be a Sylow 2-group of $G$ which contains $W_{1}$. Since $u \in W_{1}, u$ centralizes $Z\left(S_{1}\right)$. Hence we have $Z\left(S_{1}\right) \subseteq W_{1}$. There is a central involution $z_{1}$ of $Z\left(S_{1}\right)$ which is conjugate to $j_{1}$ in $C_{G}(u)$. By (7.4), $z_{1}$ is not contained in $G_{0}$. Since $z_{1}$ is not conjugate to $z$ in $C_{G}(u)$, there is an involution $w$ such that

$$
w \in C_{G}\left(z, z_{1}, u\right)
$$

and $w z$ is conjugate to either $z$ or $z_{1}$. In either case $w z$ is a central involution. Since $z$ is a central involution of $G_{0}$ and $w \in C_{G}(z)$, we have $w \in G_{0}$ by (7.3). Hence $w$ is an involution of $G_{0}$ which is a product of two commuting central involutions $w z$ and $z$. Hence $w \in C_{i}$ with $i \leqq 2$. This implies that $C_{G}(w) \subseteq G_{0}$ by (7.3) or (7.5). But $C_{G}(w)$ contains the element $z_{1}$ which is not contained in $G_{0}$. This contradiction proves the validity of (7.8).

(7.9) The group $G_{0}$ coincides with $G$.

Proof. Suppose not. There is an involution $t$ which is conjugate to $j_{2}$ of (2.2) in $G$ but is not contained in $G_{0}$. Let $z$ be an element of $C_{1}$. By (3.4), $z$ is not conjugate to $t$ in $G$. Hence there is an involution $w$ of $G$ such that

$$
w \in C_{G}(z, t)
$$

and $z w$ is conjugate to either $z$ or $t$. Since $C_{G}(z) \subseteq G_{0}, w$ is an element of $G_{0}$. The element $z w$ belongs to $C_{1} \cup C_{2}$, and $w$ is a product of commuting involutions $z$ and $z w$. Since $z \in C_{1}$, we have

$$
w \in C_{1} \cup C_{2} \cup C_{3} \text {. }
$$

By (7.3), (7.5) or (7.8), $C_{G}(w)$ is contained in $G_{0}$. But $C_{G}(w)$ contains the element $t$ which by definition lies outside $G_{0}$. This is a contradiction.

This proves (7.9), and the proof of Theorem 1 is finished. 


\section{REFERENCES}

1. E. Artin, The order of the classical simple groups, Comm. Pure Appl. Math. 8 (1955), 455-472.

2. H. Bender, Finite groups having a strongly embedded subgroup (to appear).

3. R. Brauer, On the structure of groups of finite order, Proc. Internat. Congr. Math., vol. 1, North-Holland, Amsterdam, 1954, pp. 209-217.

4. - On finite Desarguesian planes. I, II, Math. Z. 90 (1966), 117-151.

5. R. Brauer and P. Fong, A characterization of the Mathieu group $M_{12}$, Trans. Amer. Math. Soc. 122 (1966), 18-47.

6. R. Brauer and K. A. Fowler, On groups of even order, Ann. of Math. (2) 62 (1955), 565-583.

7. R. Brauer and M. Suzuki, $O n U_{3}(q)$ (unpublished).

8. R. Brauer M. Suzuki and G. E. Wall, $A$ characterization of the one-dimensional unimodular groups over finite fields, Illinois J. Math. 2 (1958), 718-745.

9. W. Burnside, Theory of groups of finite order, Cambridge, 1911.

10. W. Feit and J. G. Thompson, Solvability of groups of odd order, Pacific J. Math. 13 (1963), 775-1029.

11. P. Fong, On a characterization of $G_{2}(q)$ and $D_{4}^{2}(q)$, Sympos. Theory of Finite Groups, Benjamin, New York, 1969, pp. 25-29.

12. W. Gaschütz, Zur Erweiterungstheorie der endlichen Gruppen, Crelle 190 (1952), 93-107. 420.

13. G. Glauberman, Central elements in core-free groups, J. Algebra 4 (1966), 40398.

14. — A characterization of the Suzuki groups, Illinois J. Math. 12 (1968), 76-

15. D. Gorenstein, Finite groups the centralizers of whose involutions have normal 2complements, Canad. J. Math. 21 (1969), 335-357.

16. - Finite groups, Harper and Row, New York, 1968.

17. P. Hall, Some sufficient conditions for a group to be nilpotent, Illinois J. Math. 2 (1958), 787-801.

18. D. Held, A characterization of the alternating groups of degree eight and nine, J. Algebra 7 (1967), 218-237.

19. - A characterization of some multiply transitive permutation groups. I, Illinois J. Math. 13 (1969), 224-240.

20. - Some simple groups related to $M_{24}$, Sympos. Theory of Finite Groups, Benjamin, New York, 1969, pp. 121-124.

21. N. Ito, On a class of doubly, but not triply transitive permutation groups, Arch. Math. 18 (1967), 564-570.

22. Z. Janko, $A$ new finite simple group with abelian Sylow 2-subgroups and its characterization, J. Algebra 4 (1966), 147-136.

23. ㄴ., A characterization of the finite simple group $\mathrm{PSp}_{4}(3)$, Canad. J. Math. 19 (1967), 872-894.

24. - Some new simple groups of finite order. I, Ist. Naz. Alta Mat. Symposia Math. 1 (1968), 25-64. 1-19.

25. - A characterization of the Mathieu simple groups. I, J. Algebra 9 (1968),

26. Z. Janko and S. K. Wong, A characterization of the Higman-Sims simple group (to appear).

27. T. Kondo, On a characterization of the alternating group of degree eleven, Illinois J. Math. 13 (1969) 528-541. 
28. - On the alternating groups. I, II, III, J. Univ. Tokyo 15 (1968), 87-97; J. Math. Soc. Japan 21 (1969), 116-139; (to appear).

29. E. T. Parker, On collineations of symmetric designs, Proc. Amer. Math. Soc. 8 (1957), 350-351.

30. K. W. Phan, $A$ characterization of $L_{4}(q)$ for $q \equiv-1(\bmod 4)$, Sympos. Theory of Finite Groups, Benjamin, New York, 1969, pp. 39-42.

31. - A characterization of $U_{4}(q)$ (to appear).

32. M. Suzuki, On characterizations of linear groups. I, II, Trans. Amer. Math. Soc. 92 (1959), 191-219; III, Nagoya J. Math. 21 (1961), 159-183; IV, J. Algebra 8 (1968), 223-247; V, VI (to appear).

33. - - Two characteristic properties of $(Z T)$-groups, Osaka J. Math. 15 (1963) $143-150$.

34. - Finite groups of even order in which Sylow 2-groups are independent, Ann. of Math. (2) 80 (1964), 58-77.

35. - Finite groups in which the centralizer of any element of order 2 is 2closed, Ann. of Math. (2) 82 (1965), 191-212.

36. G. Thomas, A characterization of the groups $G_{2}\left(2^{n}\right)$, J. Algebra (to appear).

37. - A characterization of the Steinberg groups $D_{4}^{2}\left(q^{3}\right), q=2^{n}$ (to appear).

38. - A characterization of the unitary groups $\operatorname{PSU}_{5}\left(q^{2}\right), q=2^{n}$ (to appear).

39. J. G. Thompson, Nonsolvable finite groups all of whose local subgroups are solvable, Bull. Amer. Math. Soc. 74 (1968), 383-437. (Balance to appear.)

40. J. Tits, Théorème de Bruhat et sous-groupes paraboliques, C. R. Acad. Sci. Paris 254 (1962), 2910-2912.

41. - Buildings of spherical types and finite $B N$-pairs (to appear).

42. $\mathrm{H}$. Wielandt, Beziehungen zwischen den Fixpunktzahlen von Automorphismengruppen einer endlichen Gruppe, Math. Z. 73 (1960), 146-158.

43. W. J. Wong, A characterization of the alternating group of degree 8, Proc. London Math. Soc. 50 (1963), 359-383.

44. - A characterization of $\mathrm{PSp}_{4}(q), q$ odd, Sympos. Theory of Finite Groups, Benjamin, New York, 1969, pp. 31-38. Trans. Amer. Math. Soc. 139 (1969), 1-35.

45. ㄴ, A characterization of the finite projective symplectic groups $\operatorname{PSp}_{6}(q)$ (to appear).

46. $\mathrm{H}$. Yamaki, $A$ characterization of the alternating groups of degrees $12,13,14,15$, J. Math. Soc. Japan 20 (1968), 673-694.

47. - A characterization of the simple group $\mathrm{Sp}(6,2)$ (to appear).

Institute for Advanced Study, Princeton, New Jersey 08540 and

UNIVERSITY OF ILLINOIS, URBANA, ILLINOIS 61801 